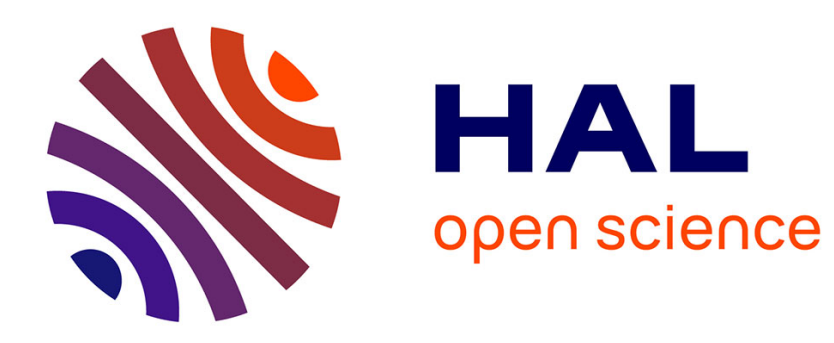

\title{
A Second-Order Bundle Method to Minimize the Maximum Eigenvalue Function
}

François Oustry

\section{To cite this version:}

François Oustry. A Second-Order Bundle Method to Minimize the Maximum Eigenvalue Function. RR-3738, INRIA. 1999. inria-00072927

\section{HAL Id: inria-00072927 \\ https://hal.inria.fr/inria-00072927}

Submitted on 24 May 2006

HAL is a multi-disciplinary open access archive for the deposit and dissemination of scientific research documents, whether they are published or not. The documents may come from teaching and research institutions in France or abroad, or from public or private research centers.
L'archive ouverte pluridisciplinaire HAL, est destinée au dépôt et à la diffusion de documents scientifiques de niveau recherche, publiés ou non, émanant des établissements d'enseignement et de recherche français ou étrangers, des laboratoires publics ou privés. 


\section{A second-order bundle method to minimize the maximum eigenvalue function}

François Oustry

$$
\mathrm{N}^{\circ} 3738
$$

Juillet 1999

THÈME 4 



\title{
A second-order bundle method to minimize the maximum eigenvalue function*
}

\author{
François Oustry \\ Thème 4 - Simulation et optimisation \\ de systèmes complexes \\ Projet Numopt
}

Rapport de recherche $\mathrm{n}^{\circ} 3738$ - Juillet 1999 - 29 pages

\begin{abstract}
In this paper we present a nonsmooth algorithm to minimize the maximum eigenvalue of matrices belonging to an affine subspace of $n \times n$ symmetric matrices. We show how a simple bundle method, the approximate eigenvalue method can be used to globalize the second-order method developed by M. L. Overton in the eighties and recently revisited in the framework of the $\mathcal{U}$-Lagrangian theory. With no additional assumption, the resulting algorithm generates a minimizing sequence. A geometrical and constructive proof is given. To prove that quadratic convergence is achieved asymptotically, some strict complementarity and non-degeneracy assumptions are needed. We also introduce a new generation of bundle methods for semidefinite programming.
\end{abstract}

Key-words: Eigenvalue optimization, semidefinite programming, convex optimization, secondorder bundle methods

(Résumé : tsvp)

* This work was initiated with a scholarship from ENSTA/DGA (France) and was pursued with a post-doctoral scholarship from INRIA and the NSF grant 655302574100-F1852 at Courant Institute of Mathematical Sciences, New York University, 251 Mercer Street, New York, NY 10012 USA. Revised version submitted to Math. Programming, June 1999. 


\section{Une méthode de faisceaux du second-ordre pour minimiser la fonction valeur propre maximale}

Résumé : Dans cet article nous présentons une méthode d'optimisation non-différentiable pour minimiser la valeur propre maximale de matrices symmétriques réelles dépendant de manière affine de paramètres réels. Nous montrons comment une méthode de faisceaux élémentaire, appelée méthode des valeurs propres approchées, peut être utilisée pour assurer la convergenve (globale) de l'algorithme du second-ordre proposé par M. L. Overton dans les années 80 et récemment revisité dans le cadre de la théorie du $\mathcal{U}$-Lagrangien. Pour montrer que l'algorithme obtenu produit une suite minimisante, nous utilisons une description géométrique du sous-différentiel de la valeur propre maximale. Pour garantir une vitesse de convergence quadratique, des hypothèses de stricte complémentarité et de non-dégénérescence sont requises. Enfin nous relions notre algorithme avec une classe plus large de méthodes de faisceaux, dédiées à la programmation sous contraintes de semidéfinie-positivité. 


\section{Introduction}

Eigenvalue optimization problems have a long history: as mentioned in [25], Lagrange had already stated in 1773 an eigenvalue optimization problem to design the shape of the strongest axially symmetric column with prescribed length, volume and boundary conditions. Yet it is only very recently that it became an independent area of research with both theoretical and practical aspects. Although the mathematical models of the underlying physical problems are generally not convex, it is notable that the area has very strong connections with convex analysis. In fact, these problems have often a composite structure with a convex component. The role of convex analysis was first emphasized by R. Bellman and K. Fan in [4]; more recently, this point of view was developed further in [15] and [25].

We consider here a basic eigenvalue optimization problem

$$
\inf _{x \in \mathbb{R}^{m}} \lambda_{1}(A(x))
$$

where $\lambda_{1}(X)$ is the largest eigenvalue of $X=A(x)$, element of $\mathcal{S}_{n}$ the space of $n \times n$ symmetric matrices and

$$
\mathbb{R}^{m} \ni x \mapsto A(x):=A_{0}+\mathcal{A} x
$$

is affine: $A_{0} \in \mathcal{S}_{n}$ and $\mathcal{A}$ is a linear operator from $\mathbb{R}^{m}$ to $\mathcal{S}_{n}$.

Existing numerical methods to solve $(P)$ can be arranged in two classes: interior-point methods and nonsmooth optimization methods. The first interior-point methods for solving $(P)$ (in the framework of semidefinite programming) were developed by Nesterov and Nemirovski [30]. With the exception of Nemirovski's projective method [31, 26], all the interior-point schemes proposed in the early 1990's (see the numerous references in [5, Chap.II, Notes and References]) were path-following or potential reduction methods. As recently explained in a clear survey by Yu. Nesterov [27], "classical" interiorpoint methods can be seen as a process to transform the initial problem into an equivalent one which can be solved "easily" thanks to an addition of structure: self-concordance is used to obtain the polynomiality of interior-point schemes [31]. A similar presentation can be done for predictorcorrector type methods using small neighborhoods; many variants of them can be found for semidefinite programming: to give only a sample we refer to $[2,38,21]$.

Our approach is quite different but, as we will see, does not exempt us from finding a tradeoff between global and local requirements, i.e., between total complexity and speed of convergence. Starting directly from problem $(P)$ itself, we will use a recent second-order theory, namely the $\mathcal{U}$ Lagrangian theory [24], to speed up the asymptotic convergence of a first-order method developed by Cullum, Donath and Wolfe [6] for a particular instance of $(P)$ ( $\mathcal{A}$ diagonal), and by Polak and Wardi [37] in a more general framework. Using the terminology of [16, Chap. XIII], the method can be seen as a Markovian dual bundle method: at each iteration an approximation of the $\varepsilon$-subdifferential is computed, via a bundling process, without using information from the previous iterations. We call it the approximate eigenvalue method. More recently a stabilization of the cutting planes algorithm was proposed in [39] and enriched in [19, 14, 23, 13] with semidefinite models of the objective function; this belongs to the class of primal bundle methods [16, Chap. XV] which are very efficient to solve large-scale problems with a moderate accuracy.

When high accuracy is needed, second-order information must be added in the model. Combining a geometrical and the Sequential Quadratic Programming approaches, a local algorithm was presented and analyzed in [10], [34], [36], [35] and [41]; in the latter two papers, a quadratic rate of convergence was obtained. Yet, in this SQP framework, the authors considered only a local analysis; issues of global convergence were not addressed.

In this paper, we present, as in [33], the second-order analysis of the maximum eigenvalue function using the $\mathcal{U}$-Lagrangian theory [24] and we show how to use the approximate eigenvalue method to globalize the second-order algorithm while preserving asymptotically a quadratic rate of convergence.

Our paper is organized as follows. We first recall some well-known results on the first-order analysis of $\lambda_{1}$. Then using simple chain rules, we derive easily a first-order analysis of the composite function $\mathrm{RR} \mathrm{n}^{\circ} 3738$ 
$f:=\lambda_{1} \circ A$. This enables us to simplify the approximate eigenvalue method developed in $\S 3$ : at a point $x$ we consider the enlargement of the subdifferential of $\lambda_{1}$ obtained with eigenvectors associated with $\varepsilon$ maximal eigenvalues; this set $\delta_{\varepsilon} f(x)$ plays the role of an approximation of the the true $\varepsilon$-subdifferential $\partial_{\varepsilon} f(x)$. By measuring the quality of this approximation, we provide an explicit $\varepsilon$-strategy to ensure global convergence of the method; this is an improvement of [32, Theorem 5.5] where the distance between the exact subdifferential of $\lambda_{1}$ at $X \in \mathcal{S}_{n}$ and its $\varepsilon$-subdifferential was considered. In $\S 4$, we present the second-order analysis of $\lambda_{1}$ using the $\mathcal{U}$-Lagrangian theory. We recall the main result of [33]: this theory provides us with a second-order development of $\lambda_{1}$ along a smooth manifold: the set $\mathcal{M}_{r}$ of matrices whose largest eigenvalue has multiplicity $r$. We derive similar results for $f$ with a so-called transversality condition. Then, in $\S 5$, we show how to use the approximate eigenvectors $(\S 2)$ to introduce some viscosity in the second-order objects presented in $\S 4$. In particular we provide a constructive characterization of the projection of a matrix $X \in \mathcal{S}_{n}$ onto the manifold $r_{\varepsilon}$ (Theorem 5.5). This results in a second-order bundle method which is globally and quadratically convergent. With no additional assumptions, a minimizing sequence is generated. Some strict complementarity and nondegeneracy assumptions are needed to guarantee the quadratic rate of convergence. In $\S 6$ using some duality, we explain how the approximate eigenvalue bundle method is related to a new generation of spectral proximal-type bundle methods in which second-order information can also be introduced. Finally we have chosen a numerical example from combinatorial optimization to illustrate a qualitative distinction between interior-point methods and second-order bundle methods: for the latter methods, superlinear convergence can be observe even when sctrict complementarity does not hold.

For the convenience of the reader an appendix explaining the notation is given at the end of the paper.

\section{First-order analysis}

In this section we recall elementary results for the maximum eigenvalue function: the subdifferentials of $\lambda_{1}$ can be characterized as exposed faces of a compact section of the cone of semidefinite matrices. Then we propose an enlargement of the subdifferential of $\lambda_{1}$ based on the computation of approximate eigenvectors and a vertical development of $\lambda_{1}$, i.e., a development of the function $\varepsilon \mapsto f_{\varepsilon}^{\prime}(x ; d)$. We derive similar results for $f:=\lambda_{1} \circ A$ using a simple chain rule. This will lead us to the main result of this section: any direction $d$ separating 0 from the chosen enlargement of $\partial f(x)$ is a "good" descent direction.

\subsection{Subdifferentials and faces}

In this paragraph we give explicit descriptions of the subdifferential and the approximate subdifferential of $\lambda_{1}$. In this analysis, a convex compact set plays a paramount role: the intersection of the cone of semidefinite matrices with the hyperplane $\left\{V \in \mathcal{S}_{n}: \operatorname{tr} V=1\right\}$,

$$
\mathcal{C}_{n}:=\left\{V \in \mathcal{S}_{n}: V \succeq 0, \operatorname{tr} V=1\right\} .
$$

The following result is well-known; the proof is easy to derive via the spectral decomposition of symmetric matrices.

Lemma 2.1 The convex set $\mathcal{C}_{n}$ is the convex hull of rank-one matrices:

$$
\mathcal{C}_{n}=\operatorname{co}\left\{q q^{T}:\|q\|=1\right\}
$$

Using Rayleigh's variational formulation

$$
\lambda_{1}(X)=\max _{q \in \mathbb{R}^{m},\|q\|=1} q^{T} X q
$$


together with Lemma 2.1, a support function formulation is obtained:

$$
\lambda_{1}(X)=\sigma_{\mathcal{C}_{n}}(X) .
$$

As in [15], we will favor the support function formulation since it will be our main tool in the analysis of $\S 2.3$. In order to describe the exposed faces of $\mathcal{C}_{n}$ (Theorem 2.3), we first establish the following technical result.

Lemma 2.2 Let $X$ and $Z$ be in $\mathcal{S}_{n}$; let $Q=\left[q_{1}, \ldots, q_{r}\right]$ be an $n \times r$ matrix whose columns form an orthonormal basis of $\operatorname{ker} X$.

(i) Then, $X Z=0$ if and only if there exists $Y \in \mathcal{S}_{r}$ such that $Z=Q Y Q^{T}$.

(ii) Assume in addition $X, Z \in \mathcal{S}_{n}^{+}$; then $\langle X, Z\rangle=0$ if and only if there exists $Y \succeq 0$ such that $Z=Q Y Q^{T}$.

Proof. [( $i)]$ We have $X Z=0$ if and only if range $Z \subset \operatorname{ker} X=\operatorname{span}\left\{q_{1}, \ldots, q_{r}\right\}$. This is equivalent to saying that $Z$ belongs to the subspace

$$
\operatorname{span}\left\{q_{i} q_{j}^{T}+q_{j} q_{i}^{T}: i, j=1, \ldots, r\right\}=Q \mathcal{S}_{r} Q^{T} .
$$

[(ii)] When $X$ and $Z$ positive semidefinite, a consequence of the Schur product Theorem [17, $\S 5.2]$ is that $\operatorname{tr} X Z=0$ if and only if $X Z=0$. Then, via $(i), Z$ has the form $Q Y Q^{T}$; it is positive semidefinite if and only if $Y \succeq 0$.

The following theorem recalls previously known geometrical descriptions of $\partial \lambda_{1}(X)$ (see [10] or [34]) and, along the lines of [15], makes an explicit link with the exposed faces of $\mathcal{C}_{n}$.

Theorem 2.3 $\quad$ (i) Let $X \in \mathcal{S}_{n}$ and let $Q_{1}$ be an $n \times r$ matrix whose columns form an orthonormal basis of $E_{1}(X)$. The face of $\mathcal{C}_{n}$ exposed by $X$ is

$$
F_{\mathcal{C}_{n}}(X)=\left\{Q_{1} Y Q_{1}^{T}: Y \in \mathcal{C}_{r}\right\}=\operatorname{co}\left\{q q^{T}:\|q\|=1, q \in E_{1}(X)\right\} ;
$$

it is the face exposed by any $X^{\prime} \in \mathcal{S}_{n}$ such that $E_{1}\left(X^{\prime}\right)=E_{1}(X)$.

(ii) The subdifferential of $\lambda_{1}$ at $X$ is

$$
\partial \lambda_{1}(X)=F_{\mathcal{C}_{n}}(X)
$$

Proof. [(i)] Realize first that

$$
\operatorname{co}\left\{q q^{T}:\|q\|=1, q \in E_{1}(X)\right\}=\left\{Q_{1} Y Q_{1}^{T}: Y \in \mathcal{C}_{r}\right\} .
$$

Indeed write any normalized vector of $E_{1}(X)$ under the form $q=Q_{1} z$, with $z \in \mathbb{R}^{r}$ and $\|z\|=1$. We get

$$
\begin{aligned}
\operatorname{co}\left\{q q^{T}:\|q\|=1, q \in E_{1}(X)\right\} & =\operatorname{co}\left\{Q_{1} z z^{T} Q_{1}^{T}: z \in \mathbb{R}^{r},\|z\|=1\right\} \\
& =Q_{1} \operatorname{co}\left\{z z^{T}: z \in \mathbb{R}^{r},\|z\|=1\right\} Q_{1}^{T},
\end{aligned}
$$

where, in view of Lemma 2.1, $\operatorname{co}\left\{z z^{T}: z \in \mathbb{R}^{r},\|z\|=1\right\}=\mathcal{C}_{r}$. Now, by definition of an exposed face, $Z \in F_{\mathcal{C}_{n}}(X)$ means

$$
Z \in \mathcal{C}_{n} \text { and }\langle X, Z\rangle=\sigma_{\mathcal{C}_{n}}(X)=\lambda_{1}(X)
$$

or equivalently,

$$
Z \in \mathcal{C}_{n} \text { and }\left\langle\lambda_{1}(X) I_{n}-X, Z\right\rangle=0 .
$$

Altogether, (4) is obtained with Lemma 2.2 and $F_{\mathcal{C}_{n}}\left(X^{\prime}\right)=F_{\mathcal{C}_{n}}(X)$ if and only if $\operatorname{ker}\left(\lambda_{1}(X) I_{n}-X\right)=$ $\operatorname{ker}\left(\lambda_{1}\left(X^{\prime}\right) I_{n}-X^{\prime}\right)$, i.e., $E_{1}(X)=E_{1}\left(X^{\prime}\right)$.

$[(i i)]$ It is well known that the subdifferential of a support function $\sigma_{\mathcal{C}_{n}}$ at a point $X$ is the exposed face of $\mathcal{C}_{n}$ exposed by $X$ [16, Example VI.3.1].

The description of the approximate subdifferential is also obtained directly from the support function formulation of $\lambda_{1}$.

$\mathrm{RR} \mathrm{n}^{\circ} 3738$ 
Theorem 2.4 For all $\varepsilon \geq 0$, we have

$$
\partial_{\varepsilon} \lambda_{1}(X)=\left\{Z \in \mathcal{C}_{n}:\langle Z, X\rangle \geq \lambda_{1}(X)-\varepsilon\right\}
$$

Proof. The approximate subdifferential of a support function is given in [16, Example XI.1.2.5]. Then (7) follows immediately. In [44] the same result is obtained via an analysis of the conjugate function of $\lambda_{1}$.

The directional derivative of $\lambda_{1}$ has an easy expression.

Theorem 2.5 For all $D \in \mathcal{S}_{n}$, we have

$$
\lambda_{1}^{\prime}(X ; D)=\lambda_{1}\left(Q_{1}^{T} D Q_{1}\right) .
$$

Proof. Use (60) and (5) to obtain

$$
\begin{aligned}
\lambda_{1}^{\prime}(X ; D) & =\sigma_{\partial \lambda_{1}(X)}(D)=\max _{Y \in \mathcal{C}_{r}}\left\langle D, Q_{1} Y Q_{1}^{T}\right\rangle \\
& =\max _{Y \in \mathcal{C}_{r}}\left\langle Q_{1}^{T} D Q_{1}, Y\right\rangle=\lambda_{1}\left(Q_{1}^{T} D Q_{1}\right)
\end{aligned}
$$

This completes the proof. This result was already established using perturbation theory in [22] and can also be found in [15], [41].

It is well-known in nonsmooth optimization that the descent property $\lambda_{1}^{\prime}(X ; D)<0$ of a direction $D$ is unstable, the function $X \mapsto \lambda_{1}^{\prime}(X ; D)$ being discontinuous. Minimization algorithms based on this mere property are usually not convergent, because descent along such directions may be numerically insufficient. One is much more interested in $\varepsilon$-descent directions, for which

$$
\lambda_{1, \varepsilon}^{\prime}(X ; D):=\sigma \partial_{\varepsilon} \lambda_{1}(X)(D)
$$

is negative. Said otherwise, these directions separate 0 from $\partial_{\varepsilon} \lambda_{1}(X)$ the continuity of $X \mapsto \lambda_{1, \varepsilon}^{\prime}(X ; D)$ [16, Theorem XI.4.1.3] guarantees the numerical efficiency of such directions. Yet the difficulty here is to get a separation algorithm, and this is the rationale for dual bundle methods [16, XIII,XIV]. This paper follows the same approach; but, instead of separating 0 from $\partial_{\varepsilon} \lambda_{1}(X)$, we use the structure of our specific problem to provide a tractable "good approximation" of the latter set.

\section{$2.2 \quad$ Enlargement of the subdifferential}

Since $\lambda_{1}$ is the support function of a convex compact set, it can be seen as an infinite-max function. Then a first idea could be to consider the enlargement proposed in [8, Chap. VI]: the convex hull of the gradients of $\varepsilon$-active functions. Here the functions are linear and it is easy to see, via (7), that the obtained enlargement is exactly the $\varepsilon$-subdifferential of $\lambda_{1}$. In [6], J. Cullum, W. E. Donath and P. Wolfe introduced a smaller set: they considered the eigenvectors of eigenvalues at a distance $\varepsilon$ from $\lambda_{1}(X)$ and the convex set generated by the associated rank-one matrices.

Definition 2.6 For $X \in \mathcal{S}_{n}$ and $\varepsilon \geq 0$ we define

- the set of indices of $\varepsilon$-largest eigenvalues

$$
I_{\varepsilon}(X):=\left\{i \in\{1, \ldots, n\}: \lambda_{i}(X)>\lambda_{1}(X)-\varepsilon\right\},
$$

- the $\varepsilon$-multiplicity of $\lambda_{1}(X): r_{\varepsilon}:=\max \left\{i: i \in I_{\varepsilon}(X)\right\}$,

- the $\varepsilon$-first eigenspace: $E_{\varepsilon}(X):=\oplus_{i \in I_{\varepsilon}(X)} E_{i}(X)$, where $E_{i}(X)$ is the eigenspace of $X$ associated with the ith eigenvalue $\lambda_{i}(X)$,

- its orthogonal complement: $F_{\varepsilon}(X):=\oplus_{i \notin I_{\varepsilon}(X)} E_{i}(X)$,

- the "spectral separation" of $\varepsilon: \Delta_{\varepsilon}(X):=\lambda_{r_{\varepsilon}}(X)-\lambda_{r_{\varepsilon}+1}(X)$. 
Pseudospectrum. The notions of approximate eigenvalues can be connected with the recent theory of pseudospectra of linear operators [43, 42]: this notion is mainly used to cope with the lack of regularity of nonsymmetric matrices; here, for symmetric matrices, a pseudospectrum can also be useful. Indeed it enables us to recover more than first-order regularity of the largest eigenvalue (precisely some local regularity of the set of approximate subgradients). For a normal matrix (in particular for a symmetric matrix) the $\varepsilon$-pseudospectrum comprises the union of the closed balls of radius $\varepsilon$ about each eigenvalue. In fact we consider here one of these $\varepsilon$-balls, the one centered at $\lambda_{1}(X)$, and we take its intersection with the spectrum of $X$. The important role played by the approximate eigenvalues justifies the wording approximate eigenvalues method.

Take now an $n \times r_{\varepsilon}$ matrix $Q_{\varepsilon}$ whose columns form an orthonormal basis of $E_{\varepsilon}(X)$. Then we define the following compact convex set:

$$
\delta_{\varepsilon} \lambda_{1}(X):=\operatorname{co}\left\{e e^{T}:\|e\|=1, e \in E_{\varepsilon}(X)\right\} .
$$

or equivalently, via Theorem 2.3,

$$
\delta_{\varepsilon} \lambda_{1}(X)=\left\{Q_{\varepsilon} Y Q_{\varepsilon}^{T}: Y \in \mathcal{C}_{r_{\varepsilon}}\right\}=F_{\mathcal{C}_{n}}\left(Q_{\varepsilon} Q_{\varepsilon}^{T}\right)=\partial \lambda_{1}\left(Q_{\varepsilon} Q_{\varepsilon}^{T}\right)
$$

This set is an outer-approximation of $\partial \lambda_{1}(X)$ and an inner-approximation of $\partial_{\varepsilon} \lambda_{1}(X)$ :

Proposition 2.7 Let $X \in \mathcal{S}_{n}$. Then for all $\varepsilon \geq 0$, we have

$$
\partial \lambda_{1}(X) \subset \delta_{\varepsilon} \lambda_{1}(X) \subset \partial_{\varepsilon} \lambda_{1}(X) .
$$

Proof. The inclusion $\partial \lambda_{1}(X) \subset \delta_{\varepsilon} \lambda_{1}(X)$ derives directly from (4) and (9). Another easy inclusion is $\delta_{\varepsilon} \lambda_{1}(X) \subset \mathcal{C}_{n}$. Take now $Z \in \delta_{\varepsilon} \lambda_{1}(X): Z=Q_{\varepsilon} Y Q_{\varepsilon}^{T}$ with $Y \in \mathcal{C}_{r_{\varepsilon}}$ implies $\langle Z, X\rangle=\left\langle Y, Q_{\varepsilon}^{T} A Q_{\varepsilon}\right\rangle \geq \lambda_{r_{\varepsilon}}$ since

$$
Q_{\varepsilon}^{T} A Q_{\varepsilon}=\operatorname{diag}\left(\lambda_{1}(X), \ldots, \lambda_{r_{\varepsilon}}(X)\right) \succeq \lambda_{r_{\varepsilon}} I_{r_{\varepsilon}}
$$

and $\operatorname{tr} Y=1$. Together with (8), we obtain $\langle Z, X\rangle \geq \lambda_{1}(X)-\varepsilon$; since $Z \in \mathcal{C}_{n}$ this means, according to (7), that $Z \in \partial_{\varepsilon} \lambda_{1}(X)$.

A crucial point consists now in quantifying the (Hausdorff) distance between our enlargement and the approximate subdifferential. One way to proceed is to get a vertical development of $\lambda_{1}$.

\subsection{Vertical development}

The notion of vertical development is presented in [33]. It will result in Theorem 2.11, which gives an explicit upper bound for the distance between the approximate subdifferential and the enlargement $\delta_{\varepsilon} \lambda_{1}(X)$. We start with some linear algebra.

Lemma 2.8 Let $U \in \mathbb{R}^{n \times n}$ be such that $U^{T} U=I_{n}$. Then, there exist $n \times n$ matrices $\left(E_{\varepsilon}, F_{\varepsilon}, \Sigma, T\right)$ such that

$$
\left\{\begin{array}{l}
\text { the columns of } E_{\varepsilon} \text { are unit vectors of } E_{\varepsilon}(X) \\
\text { the columns of } F_{\varepsilon} \text { are unit vectors of } F_{\varepsilon}(X) \\
\Sigma \text { and } T \text { are diagonal and positive semidefinite } \\
\Sigma^{2}+T^{2}=I \\
U=E_{\varepsilon} \Sigma+F_{\varepsilon} T
\end{array}\right.
$$

Proof. In [33, Lemma 5.2], we give a constructive proof of this result for $\varepsilon=0$. There is no difficulty to see that the same proof can be applied here by decomposing each column of $U$ on $E_{\varepsilon}(X) \oplus F_{\varepsilon}(X)=\mathbb{R}^{n}$.

The decomposition $\mathbb{R}^{m}=E_{\varepsilon}(X) \oplus F_{\varepsilon}(X)$ provides us with some useful relations.

$\mathrm{RR} \mathrm{n}^{\circ} 3738$ 
Lemma 2.9 Let $\left(E_{\varepsilon}, F_{\varepsilon}, \Sigma, T\right)$ be a quadruplet satisfying $(12)_{(a, b, c, d)}$ and $\Theta=\operatorname{diag}\left(\theta_{1}, \ldots, \theta_{n}\right) \in \mathcal{C}_{n}$. Then we have,

$$
\left\{\begin{array}{l}
E_{\varepsilon}^{T} A F_{\varepsilon}=F_{\varepsilon}^{T} A E_{\varepsilon}=0 \\
\lambda_{r_{\varepsilon}}(X) \leq\left\langle X,\left(E_{\varepsilon} \Theta E_{\varepsilon}^{T}\right)\right\rangle \leq \lambda_{1}(X) \\
\left\langle X, F_{\varepsilon} \Theta F_{\varepsilon}^{T}\right\rangle \leq \lambda_{r_{\varepsilon}+1}(X) \\
\operatorname{tr}(\Sigma \Theta T) \leq[\operatorname{tr}(T \Theta T)]^{1 / 2}
\end{array}\right.
$$

Proof.

[a]: The subspaces $E_{\varepsilon}(X)$ and $F_{\varepsilon}(X)$ are orthogonal and invariant by $X$. Then the columns of $X F_{\varepsilon}$ are in $F_{\varepsilon}(X)$ and they are orthogonal to the columns of $E_{\varepsilon}$. This implies $(a)$.

[b]: Since $E_{\varepsilon} \Theta E_{\varepsilon}^{T}=\sum_{i=1}^{p} \theta_{i} e_{i} e_{i}^{T}$, where the $e_{i}$ 's are the columns of $E_{\varepsilon}$, we have

$$
\left\langle X, E_{\varepsilon} \Theta E_{\varepsilon}^{T}\right\rangle=\sum_{i=1}^{p} \theta_{i} e_{i}^{T} A e_{i}
$$

Now use the fact that for all unit vectors $e \in E_{\varepsilon}(X), \lambda_{r_{\varepsilon}}(X) \leq e^{T} A e \leq \lambda_{1}(X)$, together with $\Theta=\operatorname{diag}\left(\theta_{1}, \ldots, \theta_{n}\right) \in \mathcal{C}_{n}$, to get $(b)$.

[c] Similarly to $[b]$, we have

$$
\left\langle X, F_{\varepsilon} \Theta F_{\varepsilon}^{T}\right\rangle=\sum_{i=1}^{p} \theta_{i} f_{i}^{T} A f_{i} \leq \lambda_{r_{\varepsilon}+1}(X)\left(\sum_{i=1}^{n} \theta_{i}\right)=\lambda_{r_{\varepsilon}+1}(X) .
$$

$[d]$ Note that $\Sigma \preceq I_{n}$ and $\Theta T$ is (diagonal) positive semidefinite to get

$$
\operatorname{tr}(\Sigma \Theta T) \leq \operatorname{tr}(\Theta T) .
$$

Now use $\Theta=\operatorname{diag}\left(\theta_{1}, \ldots, \theta_{n}\right) \in \mathcal{C}_{n}$ and $T=\operatorname{diag}\left(t_{1}, \ldots, t_{n}\right) \succeq 0$, together with the concavity of the square-root function, to obtain

$$
\sum_{i=1}^{n} \theta_{i} t_{i} \leq\left(\sum_{i=1}^{n} \theta_{i} t_{i}^{2}\right)^{1 / 2}
$$

In matrix notation, this means $\operatorname{tr}(\Theta T) \leq[\operatorname{tr}(T \Theta T)]^{1 / 2}$. Together with (14), this gives $(d)$.

We can now give a key result toward relating the two sets of (7) and (10).

Proposition 2.10 Let $X \in \mathcal{S}_{n}, \varepsilon \geq 0$ and $\eta \geq 0$. For all $V \in \partial_{\eta} \lambda_{1}(X)$, there exists $G_{\varepsilon} \in \delta_{\varepsilon} \lambda_{1}(X)$ and five $n \times n$ matrices $\left(E_{\varepsilon}, F_{\varepsilon}, \Sigma, T, \Theta\right)$ such that

$$
\left\{\begin{array}{l}
\left(E_{\varepsilon}, F_{\varepsilon}, \Sigma, T\right) \text { satisfies }(12)(a, b, c, d) \\
\Theta=\operatorname{diag}\left(\theta_{1}, \ldots, \theta_{n}\right) \in \mathcal{C}_{n} \\
Z=G_{\varepsilon}+\left(E_{\varepsilon} \Sigma \Theta T F_{\varepsilon}^{T}+F_{\varepsilon} \Sigma \Theta T E_{\varepsilon}^{T}\right)+\left(F_{\varepsilon} T \Theta T F_{\varepsilon}^{T}-E_{\varepsilon} T \Theta T E_{\varepsilon}^{T}\right) \\
\operatorname{tr} T \Theta T \leq \frac{\eta}{\lambda_{r_{\varepsilon}}(X)-\lambda_{r_{\varepsilon}+1}(X)}=\frac{\eta}{\Delta_{\varepsilon} \lambda_{1}(X)}
\end{array}\right.
$$

Proof. Write the spectral decomposition of $Z \in \partial_{\eta} \lambda_{1}(X)$ : there exists $U \in \mathbb{R}^{n \times n}$, such that $U^{T} U=I_{n}$ and a diagonal matrix $\Theta$ such that $Z=U \Theta U^{T}$. In view of (7), we have $\Theta \in \mathcal{C}_{n}$. Then, apply Lemma 2.8: $U=E_{\varepsilon} \Sigma+F_{\varepsilon} T$ where $\left(E_{\varepsilon}, F_{\varepsilon}, \Sigma, T\right)$ satisfies $(12)_{(a, b, c, d)}$. Substituting this in the spectral decomposition of $Z$, we obtain

$$
Z=G_{\varepsilon}+\left(E_{\varepsilon} \Sigma \Theta T F_{\varepsilon}^{T}+F_{\varepsilon} T \Theta \Sigma E_{\varepsilon}^{T}\right)+\left(F_{\varepsilon} T \Theta T F_{\varepsilon}^{T}-E_{\varepsilon} T \Theta T E_{\varepsilon}^{T}\right),
$$

where $G_{\varepsilon}:=E_{\varepsilon} \Theta E_{\varepsilon}^{T}=\sum_{i=1}^{p} \theta_{i} e_{i} e_{i}^{T}$ and the $e_{i}$ 's are unit vectors of $E_{\varepsilon}(X)$. According to (10), this means $G_{\varepsilon} \in \delta_{\varepsilon} \lambda_{1}(X)$. Then $(a, b, c)$ are satisfied. In order to prove $(d)$, take the scalar product of $X$ with the left- and right-hand side of (16) and use $(13)_{(a, b, c, d)}$ to obtain

$$
\lambda_{1}(X)+\left(\lambda_{r_{\varepsilon}+1}(X)-\lambda_{r_{\varepsilon}}(X)\right) \operatorname{tr}(T \Theta T) \geq\langle X, Z\rangle .
$$


Since $Z \in \partial_{\eta} \lambda_{1}(X)$, we have together with (7), $\langle X, Z\rangle \geq \lambda_{1}(X)-\eta$. This enables us to complete the proof.

The following result says that $\delta_{\varepsilon} \lambda_{1}(X)$ is a good approximation of $\partial_{\eta} \lambda_{1}(X)$ for $\eta$ small enough, depending on the spectral separation of Definition 2.6.

Theorem 2.11 For all $\varepsilon \geq 0, \eta \geq 0$ and $D \in \mathcal{S}_{n}$, we have

$$
\lambda_{1, \eta}^{\prime}(A ; D) \leq \sigma_{\delta_{\varepsilon} \lambda_{1}(X)}(D)+\rho(\eta, \varepsilon)\|D\|,
$$

or equivalently,

$$
\partial_{\eta} \lambda_{1}(X) \subset \delta_{\varepsilon} \lambda_{1}(X)+B(0, \rho(\eta, \varepsilon))
$$

where $\rho(\eta, \varepsilon):=\left(\frac{2 \eta}{\Delta_{\varepsilon}(X)}\right)^{1 / 2}+\left(\frac{2 \eta}{\Delta_{\varepsilon}(X)}\right)$.

Proof. Let $\varepsilon \geq 0, \eta \geq 0, D \in \mathcal{S}_{n}$ and $Z \in \partial_{\eta} \lambda_{1}(X)$. From $\left(_{(15)}\right.$, we obtain

$$
\langle Z, D\rangle=\left\langle G_{\varepsilon}, D\right\rangle+\left\langle\Sigma \Theta T, E_{\varepsilon}^{T} D F_{\varepsilon}+F_{\varepsilon}^{T} D E_{\varepsilon}\right\rangle+\left\langle T \Theta T, F_{\varepsilon}^{T} D F_{\varepsilon}-E_{\varepsilon}^{T} D E_{\varepsilon}\right\rangle .
$$

Let us bound each of the three terms from above. First, $G_{\varepsilon} \in \delta_{\varepsilon} \lambda_{1}(X)$ implies

$$
\left\langle G_{\varepsilon}, D\right\rangle \leq \sigma_{\delta_{\varepsilon} \lambda_{1}(X)}(D) .
$$

Then, denoting $(\Sigma \Theta T)=\operatorname{diag}\left(\sigma_{1} \theta_{1} t_{1}, \ldots, \sigma_{n} \theta_{n} t_{n}\right)$, we have

$$
\left\langle\Sigma \Theta T, E_{\varepsilon}^{T} D F_{\varepsilon}+F_{\varepsilon}^{T} D E_{\varepsilon}\right\rangle=\sum_{i=1}^{n} \sigma_{i} \theta_{i} t_{i}\left[\left\langle D, e_{i} f_{i}^{T}+f_{i} e_{i}^{T}\right\rangle\right] .
$$

Now, use the Cauchy-Schwarz inequality, together with $\left\|e_{i} f_{i}^{T}+f_{i} e_{i}^{T}\right\|=\sqrt{2}$, to get

$$
\left\langle\Sigma \Theta T, E_{\varepsilon}^{T} D F_{\varepsilon}+F_{\varepsilon}^{T} D E_{\varepsilon}\right\rangle \leq \sqrt{2}\|D\| \operatorname{tr}(\Sigma \Theta T) .
$$

Similarly we have for the last term

$$
\begin{aligned}
\left\langle T \Theta T, F_{\varepsilon}^{T} D F_{\varepsilon}-E_{\varepsilon}^{T} D E_{\varepsilon}\right\rangle & =\sum_{i=1}^{n} \theta_{i} t_{i}^{2}\left[\left\langle D, f_{i} f_{i}^{T}-e_{i} e_{i}^{T}\right\rangle\right] \\
& \leq \operatorname{tr}(T \Theta T)\|D\|\left(\left\|f_{i} f_{i}^{T}\right\|+\left\|e_{i} e_{i}^{T}\right\|\right) \\
& \leq 2 \operatorname{tr}(T \Theta T)\|D\|,
\end{aligned}
$$

since $\left\|f_{i} f_{i}^{T}\right\|=\left\|e_{i} e_{i}^{T}\right\|=1$. Putting together (19), (20), (13)-(d) and (21), we get

$$
\langle Z, D\rangle \leq \sigma_{\delta_{\varepsilon}} \lambda_{1}(X)(D)+\sqrt{2}\|D\|(\operatorname{tr}(T \Theta T))^{1 / 2}+2\|D\| \operatorname{tr}(T \Theta T) .
$$

Together with $(15)_{(d)}$, we obtain $(17) ;(18)$ is the geometrical form of (17).

We will use this result in a simplified form.

Corollary 2.12 Let $X \in \mathcal{S}_{n}, \varepsilon \geq 0$ and $\eta \in\left[0, \frac{\Delta_{\varepsilon}(X)}{2}\right]$. Then for all $D \in \mathcal{S}_{n}$, we have

$$
\lambda_{1, \eta}^{\prime}(X ; D) \leq \sigma_{\delta_{\varepsilon} \lambda_{1}(X)}(D)+\left(\frac{8 \eta}{\Delta_{\varepsilon}(X)}\right)^{1 / 2}\|D\| .
$$

Proof. Since $\eta \leq \frac{\Delta_{\varepsilon}(X)}{2}$, we have $0 \leq \frac{2 \eta}{\Delta_{\varepsilon}(X)} \leq\left(\frac{2 \eta}{\Delta_{\varepsilon}(X)}\right)^{1 / 2}$ which, together with (17), gives (23).

Finally, we show we have s simple expression for the support function of $\delta_{\varepsilon} \lambda_{1}(X)$ : it is the largest eigenvalue of an $r_{\varepsilon} \times r_{\varepsilon}$ symmetric matrix.

Proposition 2.13 Let $X \in \mathcal{S}_{n}$. Then for all $\varepsilon \geq 0$ and $D \in \mathcal{S}_{n}$, we have

$$
\sigma_{\delta_{\varepsilon} \lambda_{1}(X)}(D)=\lambda_{1}\left(Q_{\varepsilon}^{T} D Q_{\varepsilon}\right)
$$

Proof. The set $\delta_{\varepsilon} \lambda_{1}(X)$ has the same structure as $\partial \lambda_{1}(X)$; the proof is then similar to that of Theorem 2.5.

In the following paragraph we extend these results to the composite function $f:=\lambda_{1} \circ A$. $\mathrm{RR} \mathrm{n}^{\circ} 3738$ 


\subsection{Composition with an affine mapping}

We recall that $\mathcal{A}$ denotes the linear part of $A(\cdot)$ and $\mathcal{A}^{*}$ its adjoint. First-order composition is based on the following elementary chain rule.

Proposition 2.14 For $x \in \mathbb{R}^{m}$ and $\varepsilon \geq 0$,

$$
\partial_{\varepsilon} f(x)=\mathcal{A}^{*} \partial_{\varepsilon} \lambda_{1}(A(x)) .
$$

Proof. This is a straightforward application of the chain rule given in [16, Theorem XI.3.2.1].

Then an enlargement of $\partial f(x)$ is the following convex set:

$$
\delta_{\varepsilon} f(x):=\mathcal{A}^{*} \delta_{\varepsilon} \lambda_{1}(A(x))
$$

and its associated support function

$$
\tilde{f}_{\varepsilon}^{\prime}(x ; d):=\sigma_{\delta_{\varepsilon} f(x)}(d) .
$$

Here we use the notation $\tilde{f}_{\varepsilon}^{\prime}(x ; d)$ to emphasize the analogy with the approximate directional derivative of $f$ at $x$ :

$$
f_{\varepsilon}^{\prime}(x ; d):=\sigma_{\partial_{\varepsilon} f(x)}(d) .
$$

Applying the linear mapping $\mathcal{A}^{*}$ in $(11)$, it comes

$$
\partial f(x) \subset \delta_{\varepsilon} f(x) \subset \partial_{\varepsilon} f(x), \quad[\text { geometric form }]
$$

or equivalently

$$
\left.f^{\prime}(x ; d) \leq \tilde{f}_{\varepsilon}^{\prime}(x ; d) \leq f_{\varepsilon}^{\prime}(x ; d), \quad \text { [analytic form }\right]
$$

The quality of this approximation is derived from inequality (23): for all $\varepsilon \geq 0, \eta \in\left[0, \Delta_{\varepsilon}(A(x))\right]$ and $d \in \mathbb{R}^{m}$,

$$
f_{\eta}^{\prime}(x ; d) \leq \tilde{f}_{\varepsilon}^{\prime}(x ; d)+\left(\frac{8 \eta}{\Delta_{\varepsilon}(A(x))}\right)^{1 / 2}\|\mathcal{A} d\| \leq \tilde{f}_{\varepsilon}^{\prime}(x ; d)+\left(\frac{8 \eta}{\Delta_{\varepsilon}(A(x))}\right)^{1 / 2} \kappa\|d\|,
$$

where $\kappa:=\sup _{\|x\|=1}\|\mathcal{A}(x)\|$ is the largest singular value of $\mathcal{A}$.

Furthermore, it is straightforward from $(24)$ and $(26)$ that $\tilde{f}_{\varepsilon}^{\prime}(x ; d)$ is also a maximum eigenvalue:

$$
\tilde{f}_{\varepsilon}^{\prime}(x ; d)=\lambda_{1}\left(Q_{\varepsilon}^{T}(\mathcal{A} d) Q_{\varepsilon}\right) .
$$

In particular for $\varepsilon=0$, we have

$$
\tilde{f}_{0}^{\prime}(x ; d)=f^{\prime}(x ; d)=\lambda_{1}\left(Q_{1}(\mathcal{A} d) Q_{1}^{T}\right) .
$$

Ideally we would like to choose $\varepsilon>0$ and find a so-called direction $d$ of $\varepsilon$-descent.

Lemma 2.15 ([16, Lemma XIII.1.2.3]) Suppose that $d \in \mathbb{R}^{m}$ is a direction of $\varepsilon$-descent: $f_{\varepsilon}^{\prime}(x ; d)<$ 0 . Then the set of all $t>0$ such that $f(x+t d)<f(x)-\varepsilon$ forms a non empty interval.

The difficulty here is that $\partial_{\varepsilon} f(x)$ is so rich that computing its support function $f^{\prime}(x ; d)$ for a given direction $d$ or a fortiori looking for the best $\varepsilon$-descent direction $\operatorname{Argmin}_{\|d\|=1} f^{\prime}(x ; d)$ seems to be as expensive as the original problem $(P)$. Therefore instead of working with $\partial_{\varepsilon} f(x)$ we deal with its inner approximation $\delta_{\varepsilon} f(x)$. Equality (30) quantifies the effort needed to evaluate $\tilde{f}_{\varepsilon}^{\prime}(x ; d)$ : it requires the computation of the largest eigenvalue of an $r_{\varepsilon} \times r_{\varepsilon}$ matrix. We can now specify to what extent a direction $d \in \mathbb{R}^{m}$ satisfying at $x \in \mathbb{R}^{m}$

$$
\tilde{f}_{\varepsilon}^{\prime}(x ; d)<0,
$$

is a "good" descent direction. 
Theorem 2.16 Let $x \in \mathbb{R}^{m}, \varepsilon \geq 0$ and $d \in \mathbb{R}^{m}$ be such that (32) holds. Then

(i) $d$ is a direction of $\eta(x, \varepsilon)$-descent, where

$$
\eta(x, \varepsilon):=\left[\frac{\tilde{f}_{\varepsilon}^{\prime}(x ; d)}{4 \kappa\|d\|}\right]^{2} \Delta_{\varepsilon}(A(x)) .
$$

(ii) In addition, assume there exist $\omega \in[0,1], \delta>0$ and $\mu>0$ such that

$$
\tilde{f}_{\varepsilon}^{\prime}(x ; d) \leq-\omega\|d\|^{2},
$$

$\|d\| \geq \delta$ and $\Delta_{\varepsilon}(A(x)) \geq \mu ;$ then

$$
\eta(x, \varepsilon) \geq\left[\frac{\omega \delta}{4 \kappa}\right]^{2} \mu
$$

Proof. It is straightforward from (29).

Remark 2.17 Let $g \in \delta_{\varepsilon} f(x)$ be such that $d=-g$ satisfies (33) for $\omega=1$; then $g=\operatorname{proj}_{\delta_{\varepsilon}} f(x) 0$. When $\omega \in] 0,1], g$ is an approximation of this projection. In $\S 3.1$, we present a separation algorithm to obtain such directions.

\section{First-order algorithm}

We describe here an iterative process to compute $\eta$-descent directions using the information stored in $\delta_{\varepsilon} f(x), \eta$ and $\varepsilon$ being related as in Theorem 2.16. Then the step-length is determined with a (finite) dichotomous line-search for $\eta$-descent. The approximate eigenvalue algorithm and its convergence analysis complete the section.

\subsection{Projection problem}

The problem we want to solve is

$$
\min _{g \in \delta_{\varepsilon} f(x)}\|g\|^{2} .
$$

This problem is a quadratic optimization problem over the cone of positive semidefinite matrices. Indeed, in view of (26) and (10), program (35) is equivalent to

$$
\left\{\begin{array}{l}
\min \left\|\mathcal{A}^{*}\left(Q_{\varepsilon} Y Q_{\varepsilon}^{T}\right)\right\|^{2} \\
Y \succeq 0 \\
\operatorname{tr} Y=1
\end{array}\right.
$$

In [35], a similar projection problem is encountered. The authors adopt the following approach: instead of projecting onto $\delta_{\varepsilon} f(x)$, they project onto aff $\delta_{\varepsilon} f(x)$, i.e., the constraint $Y \succeq 0$ is replaced by $Y \in \mathcal{S}_{r_{\varepsilon}}$. This leads them to a quadratic problem with linear equality constraints which can be solved with classical (and efficient) techniques. Yet this approach has a major drawback: when the minimizer is not positive semidefinite, we have no interpretation for the resulting projection; one has to escape from the current iteration and to change the multiplicity $r_{\varepsilon}$.

Here we compute an approximation $g$ of $\operatorname{proj}_{\delta_{\varepsilon}} f(x) 0$ : we require

$$
\tilde{f}_{\varepsilon}^{\prime}(x ;-g) \leq-\omega\|g\|^{2},
$$

$\mathrm{RR} \mathrm{n}^{\circ} 3738$ 
where $\omega \in] 0,1]$ is a tolerance that controls the proximity of $g$ from the projection $\operatorname{proj}_{\delta_{\varepsilon}} f(x)$ (see Remark 2.17). The algorithm we present here is essentially the one proposed by J. Cullum, W.E. Donath and P. Wolfe in [6]. It is an instance of a general scheme, called the support-black box method in $[16, \S$ IX.3] to minimize a quadratic form on a convex set. This algorithm is in fact a separation algorithm that was first exposed in [12]. Nowadays two other alternatives are proposed in the context of proximal type bundle method: one consists in calling an interior-point method subroutine [14] and a more recent one proposes to compute an explicit solution in a well chosen metric [13].

SUPPORT-BLACK BOX METHOD

Step 0. Set $l=1$ and $s=s_{1} \in \delta_{\varepsilon} f(x)$.

Step 1. Compute $d_{l}=-\operatorname{proj}_{P_{l}} 0$, where $P_{l}=\operatorname{co}\left\{s_{1}, \ldots, s_{l}\right\}$.

Step 2. Compute $s_{l+1} \in \delta_{\varepsilon} f(x)$ such that

$$
s_{l+1}^{T} d_{l}=\sigma_{\delta_{\varepsilon}} f(x)\left(d_{l}\right) .
$$

Step 3. (Stopping criterion). If $\sigma_{\delta_{\varepsilon}} f(x)\left(d_{l}\right) \leq-\omega\left\|d_{l}\right\|^{2}$ then STOP.

Replace $l$ by $l+1$ and return to Step 1 .

Note that $s_{l+1}$ in Step 2 has the form $\mathcal{A}^{*} u u^{T}$ where $u$ is a unit eigenvector associated with $\lambda_{1}\left(Q_{\varepsilon}^{T}\left(\mathcal{A} d_{l}\right) Q_{\varepsilon}\right)$. During the separation process a bundle of $\varepsilon$-subgradients $\left\{s_{1}, \ldots, s_{k}\right\}$ is generated; in that sense the first order method presented in this section is a bundle method.

The convergence of the support-black box method is investigated in detail in $[16, \S \mathrm{IX} .3]$. In particular, we have the following properties.

Proposition 3.1 The support-black box method at $x \in \mathbb{R}^{m}$ with $\left.\omega \in\right] 0,1[$ converges in a finite number of steps. Assume that the $\varepsilon$-Strict Complementarity condition holds at $x \in \mathbb{R}^{m}$,

$$
(S C)_{\varepsilon} \quad \operatorname{proj}_{\delta_{\varepsilon}} f(x)=\operatorname{ri} \delta_{\varepsilon} f(x) .
$$

Then finite convergence is obtained also if $\omega=1$.

Proof. Set $g_{\varepsilon}:=\operatorname{proj}_{\delta_{\varepsilon}} f(x)$. Then the results are directly derived from Theorem IX.3.3.3 and Proposition IX.3.3.4 in [16].

Remark 3.2 The complexity of the support-black box method is not explicitly known. It seems that the number of steps will depend on the condition number of the linear operator

$$
\mathcal{S}_{r_{\varepsilon}} \ni Y \mapsto \mathcal{K}_{\varepsilon}(Y):=\mathcal{A}^{*}\left(Q_{\varepsilon} Y Q_{\varepsilon}^{T}-\frac{1}{r_{\varepsilon}}\left(Q_{\varepsilon} Q_{\varepsilon}^{T}\right) .\right.
$$

Requiring that $\mathcal{K}_{\varepsilon}$ is not singular was already a condition introduced in [35] and was the first apparition of the notion of transversality in semidefinite programming although it was not named as such. The connection is clearly established in [41].

\subsection{Line-search}

Let $x \in \mathbb{R}^{m}$ and $d \in \mathbb{R}^{m}$ be produced by the support-black box method of $\S 3.1$, so that $\tilde{f}_{\varepsilon}^{\prime}(x ; d)<0$. Then, according to Theorem 2.16, the objective function can be decreased by a positive number $\eta(x, \varepsilon)$. The problem we consider is now: find $t>0$ such that

$$
f(x+t d) \leq f(x)-\eta(x, \varepsilon) .
$$


When assumptions of Theorem 2.16 (ii) hold, a simple line-search for $\eta$-descent can be presented. We expose here a line-search based on a dichotomous scheme controlled by a $\eta$-descent stopping criterion. For an advanced implementation we refer to [16, Remark XIII.2.1.2].

LINE-SEARCH

Step 0. Set $t_{L}=0, t_{R}=+\infty$ and $t_{0}=1$.

Step 1 (work). Obtain $q(t):=f(x+t d)$ and $q_{+}^{\prime}(t):=f^{\prime}(x+t d, d)$ using (31).

Step 2 ( $\eta$-descent test). If (38) holds stop.

Step 3 (Dichotomous search). If $q_{+}^{\prime}(t)>0$ set $t_{R}:=t$; else set $t_{L}=t$. Compute $t=\frac{t_{L}+t_{R}}{2}$.

Theorem 3.3 Let $x \in \mathbb{R}^{m}, \varepsilon>0$ and $d \in \mathbb{R}^{m}$ be satisfying the assumptions of Theorem 2.16(ii). Then the line-search for $\eta(x, \varepsilon)$-descent stops after a finite number of steps.

Proof. From Theorem $2.16(i i)$, the quantity $\eta(x, \varepsilon)$ is strictly positive. Use now Theorem $2.16(i)$, together with Lemma 2.15: $\left\{t: \in \mathbb{R}_{+}: f(x+t d) \leq f(x)-\eta(x, d)\right\}$ is a non empty interval. Therefore the dichotomous scheme will detect one of its elements after a finite number of iterations.

\subsection{The approximate eigenvalues algorithm}

To get a simple convergence analysis, the first-order algorithm we present here will use the following $\varepsilon$-strategy (see Figure 3.3). Choose first a tolerance $\bar{\varepsilon}>0$. Then define at $x \in \mathbb{R}^{m}$

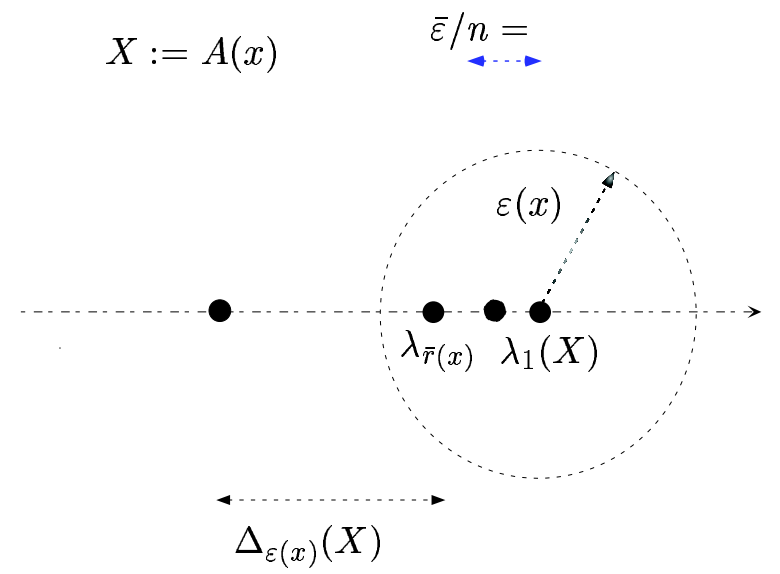

Figure 1: $\varepsilon$-strategy

$$
\left\{\begin{array}{l}
R_{\bar{\varepsilon}}(x):=\left\{r \in\{1, \ldots, n-1\}: \lambda_{r}(A(x))-\lambda_{r+1}(A(x)) \geq \frac{\bar{\varepsilon}}{n}\right\} \\
\bar{r}(x):= \begin{cases}\left\{r: r \in \min R_{\bar{\varepsilon}}(x)\right\} & \text { if } R_{\bar{\varepsilon}}(x) \neq \emptyset \\
n & \text { otherwise }\end{cases} \\
\varepsilon(x):= \begin{cases}f(x)-\lambda_{\bar{r}(x)}(A(x))+\frac{\bar{\varepsilon}}{2 n} & \text { if } R_{\bar{\varepsilon}}(x) \neq \emptyset \\
\bar{\varepsilon} & \text { otherwise. }\end{cases}
\end{array}\right.
$$

Here, $\bar{\varepsilon}$ is the final tolerance: Algorithm 3.4 below is aimed at minimizing $f$ within a tolerance $\bar{\varepsilon}$. With reference to Definition 2.6, observe that $\bar{r}(x)$ is the $\varepsilon(x)$-multiplicity of $\lambda_{1}(A(x))$. If $R_{\bar{\varepsilon}}(x) \neq \emptyset$, the spectral separation of $\varepsilon(x)$ is larger than $\frac{\bar{\varepsilon}}{n}$. Moreover we have $\lambda_{r}(A(x))-\lambda_{r+1}(A(x))<\frac{\bar{\varepsilon}}{n}$, for $r=1, \ldots, \bar{r}(x)-1$, when $\bar{r}(x)>1$.

$\mathrm{RR} \mathrm{n}^{\circ} 3738$ 


\section{Algorithm 3.4}

Step 0 (Initialization). Choose the tolerances $\delta>0, \bar{\varepsilon}>0, \omega \in] 0,1\left[\right.$; initialize $x:=x_{0} \in \mathbb{R}^{m}$.

Step 1 (Separation). Set $\varepsilon:=\varepsilon(x)$ and compute $d \in-\delta_{\varepsilon} f(x)$ satisfying (33) with the support-black box method.

Step 2 (Stopping criterion). If $\|d\| \leq \delta$ stop.

Step 3 (Line-search). Compute $t$ such that

$$
f(x+t d) \leq f(x)-\eta(x, \varepsilon),
$$

with the Safeguarded Armijo Line-Search.

Step 5 (Update). Replace $x$ by $x+t d$; return to Step 1 .

To ensure that our problem $(P)$ makes sense, we assume that $f$ is bounded from below. We have:

Lemma 3.5 The function $f$ is bounded from below if and only if

or equivalently,

$$
0 \in \mathcal{A}^{*}\left(\mathcal{C}_{n}\right)
$$

$$
(\text { range } \mathcal{A})^{\perp} \cap \mathcal{S}_{n}^{+} \neq\{0\} .
$$

Proof. From convex duality [16, Chap. X], $-f^{*}(0)=\inf _{x \in \mathbb{R}^{m}} f(x)$, where $f^{*}$ is the conjugate function of $f$; as shown in [44], we have

$$
-f^{*}(0)=\sup \left\{\left\langle Z, A_{0}\right\rangle: Z \in \mathcal{C}_{n} \cap \operatorname{ker} \mathcal{A}^{*}\right\} .
$$

Then $f$ is bounded from below if and only if $\mathcal{C}_{n} \cap \operatorname{ker} A^{*} \neq \emptyset$, which is clearly equivalent to $(i)$ and (ii). Note that $(i i)$ can be directly obtained from [26].

This leads us to the following result.

Lemma 3.6 For all $x \in \mathbb{R}^{m}$, the parameter $\varepsilon(x)$ of (39) is not greater than $\bar{\varepsilon}$. Therefore

$$
\delta_{\varepsilon(x)} f(x) \subset \partial_{\bar{\varepsilon}} f(x) .
$$

As a result, if $f$ is bounded from below, we have

$$
\begin{array}{ll}
(i) & \Delta_{\varepsilon(x)}(A(x)) \geq \frac{\bar{\varepsilon}}{n}, \\
(\text { ii }) \quad \text { or } \quad & 0 \in \delta_{\varepsilon(x)} f(x) .
\end{array}
$$

Proof. Take $x \in \mathbb{R}^{m}$ and consider the two following cases.

$\left[R_{\bar{\varepsilon}}(x) \neq \emptyset\right]$ : by construction of $\varepsilon(x)(39),(i)$ holds: $\Delta_{\varepsilon(x)}(A(x)) \geq \frac{\bar{\varepsilon}}{n}$. Furthermore

$$
\varepsilon(x)=f(x)-\lambda_{\bar{r}(x)}(A(x))+\frac{\bar{\varepsilon}}{2 n}<\left(\bar{r}(x)-\frac{1}{2}\right) \frac{\bar{\varepsilon}}{n} \leq \bar{\varepsilon} ;
$$

together with (28) and the fact that the set-valued function $\varepsilon \mapsto \partial_{\varepsilon} f(x)$ is not decreasing, this gives (40).

$\left[R_{\bar{\varepsilon}}=\emptyset\right]$ : we have $\bar{r}(x)=n$ and $\varepsilon(x)=\bar{\varepsilon}$. Hence (40) still holds. Then from (10) and (26), we have $\delta_{\varepsilon(x)} f(x)=\mathcal{A}^{*}\left(\mathcal{C}_{n}\right)$. On the other hand, we know from Lemma 3.5 that $f$ bounded means $0 \in \mathcal{A}^{*}\left(\mathcal{C}_{n}\right)$. This implies $(i i)$.

Remark 3.7 In practice we will use $\varepsilon$ bigger than $\varepsilon(x)$ : at each iteration $k$ we choose $\varepsilon_{k}$ in $\left[0, \max \left\{\varepsilon\left(x_{k}\right), \theta^{k} \varepsilon_{0}\right\}[\right.$ such that $\Delta_{\varepsilon_{k}}\left(x_{k}\right)$ is maximized, where $\left.\theta \in\right] \frac{1}{2}, 1[$ is a given parameter which forces the convergence of $\varepsilon_{k}$ towards $\bar{\varepsilon}$. 
We are now in a position to prove finite convergence of Algorithm 3.4 towards an approximate solution of $(P)$.

Theorem 3.8 Assume that $f$ is bounded from below. Then Algorithm 3.4 stops after a finite number of iterations, yielding $\bar{x}$ satisfying the approximate minimality condition

$$
f(y) \geq f(\bar{x})-\bar{\varepsilon}-\delta\|y-\bar{x}\| \text { for all } y \in \mathbf{R}^{m}
$$

Proof. While $\|d\|>\delta$, we have $0 \notin \delta_{\varepsilon(x)} f(x)$ and according to Lemma 3.6, $\Delta_{\varepsilon(x)}(A(x)) \geq \frac{\bar{\varepsilon}}{n}$. Together with (34), this gives us $f(x)-f(x+t d) \geq \eta(x, \varepsilon) \geq\left[\frac{m \delta}{4 \kappa}\right]^{2} \frac{\bar{\varepsilon}}{n}$. Then Algorithm 3.4 must stop before $N$ steps, where $N$ is the first integer satisfying

$$
f\left(x_{0}\right)-N\left[\frac{m \delta}{4 \kappa}\right]^{2} \frac{\bar{\varepsilon}}{n} \leq-f^{*}(0) .
$$

\section{Second-order analysis}

As with the first-order analysis, a convenient approach consists in studying the second-order behavior of $\lambda_{1}$ and then deriving that for the composite function $f=\lambda_{1} \circ A$ by chain rules. Yet, even though their formulation is simple, chain rules are not easy to obtain here; as explained in [33], we need to introduce a geometrical condition to get them.

\subsection{The $\mathcal{U}$-Lagrangian of $\lambda_{1}$}

For a presentation of the $\mathcal{U}$-Lagrangian theory in a more general framework we refer to [24]. The second-order analysis we present here starts with the following idea: consider at $X \in \mathcal{S}_{n}$, the largest subspace where $\lambda_{1}^{\prime}(X ; \cdot)$ is linear.

Definition 4.1 At $X \in \mathcal{S}_{n}$, we define

$$
\mathcal{U}(X):=\left\{U \in \mathcal{S}_{n}: \lambda_{1}^{\prime}(X ; U)+\lambda_{1}^{\prime}(X ;-U)=0\right\}
$$

and $\mathcal{V}(X):=\mathcal{U}(X)^{\perp}$

The subspaces $\mathcal{U}(X)$ and $\mathcal{V}(X)$ are also characterized as follows.

Proposition $4.2([24])$ Let $X \in \mathcal{S}_{n}$.

(i) For any $G \in \operatorname{ri} \partial \lambda_{1}(X), \mathcal{U}(X)$ and $\mathcal{V}(X)$ are respectively the normal and tangent cones to $\partial \lambda_{1}(X)$ at $G$.

(ii) $\mathcal{U}(X)$ and $\mathcal{V}(X)$ are respectively the subspaces orthogonal and parallel to aff $\partial \lambda_{1}(X)$.

A first attempt to reach second order could consist in introducing the function induced by $\lambda_{1}$ in $\mathcal{U}(X)$. Yet, proceeding this way we would miss a major fact: a good model of $\lambda_{1}$ must consider the local behavior of all "active constraints" at $X$. At this stage geometry can help: it suggests fixing the multiplicity (i.e., the "activity") of $\lambda_{1}$; this point of view is the one adopted in [36]. The "surface of activity" is defined as

$$
\mathcal{M}_{r}:=\left\{M \in \mathcal{S}_{n}: \lambda_{1}(M)=\ldots=\lambda_{r}(M)>\lambda_{r+1}(M)\right\} .
$$

Reference [3] is usually proposed to prove the smoothness of $\mathcal{M}_{r}$. In fact, it can be obtained as a simple consequence of the Constant Rank Theorem [40, Chap. III $\S 9$. This gives us a geometrical interpretation of the subspaces $\mathcal{U}(X)$ and $\mathcal{V}(X)$.

$\mathrm{RR} \mathrm{n}^{\circ} 3738$ 
Theorem 4.3 ([33, Corollary 4.8]) Let $X \in \mathcal{M}_{r}$. The subspaces $\mathcal{U}(X)$ and $\mathcal{V}(X)$ are respectively the tangent and normal spaces to $\mathcal{M}_{r}$ at $X$ :

$$
\begin{aligned}
& \mathcal{U}(X)=\left\{U \in \mathcal{S}_{n}: Q_{1}^{T} U Q_{1}-\frac{1}{r}\left(\operatorname{tr} Q_{1}^{T} U Q_{1}\right) I_{r}=0\right\} \\
& \text { and } \mathcal{V}(X)=\left\{Q_{1} Y Q_{1}^{T}: Y \in \mathcal{S}_{r},\left\langle Y, I_{r}\right\rangle=0\right\}
\end{aligned}
$$

The $\mathcal{U}$-Lagrangian is a convex function which identifies locally the "ridge" of $\lambda_{1}$.

Definition 4.4 Let $X \in \mathcal{S}_{n}$ and $G \in \partial \lambda_{1}(X)$. The $\mathcal{U}$-Lagrangian of $\lambda_{1}$ at the primal-dual pair $(X, G)$ is the function

$$
\mathcal{U}(X) \ni U \mapsto L_{\mathcal{U}}(X, G ; U):=\min _{V \in \mathcal{V}(X)} \lambda_{1}(X+U+V)-\langle G, V\rangle .
$$

We define also the associated set of minimizers

$$
V(X, G ; U)=\operatorname{Argmin}_{V \in \mathcal{V}(X)} \lambda_{1}(X+U+V)-\langle G, V\rangle .
$$

The following theorem is established in [24] for any finite valued convex function, we express it in our specific context.

Theorem 4.5 Let $(X, G) \in \mathcal{S}_{n} \times \partial \lambda_{1}(X)$. Then, we have

(i) the function $L_{\mathcal{U}}(X, G ; \cdot)$ is well-defined and convex over $\mathcal{U}(X)$.

Assume, in addition, that $G \in \operatorname{ri} \partial \lambda_{1}(X)$. Then,

(ii) for all $U \in \mathcal{U}(X), V(X, G ; U)$ is a nonempty compact convex set which satisfies

$$
\sup _{V \in V(X, G ; U)}\|V\|=o(\|U\|) .
$$

(iii) In particular, at $U=0$, we have $V(U)=\{0\}, L_{\mathcal{U}}(X, G ; 0)=\lambda_{1}(X)$ and $\nabla L_{\mathcal{U}}(X, G ; 0)=$ $\operatorname{proj}_{\mathcal{U}(X)} G$ exists.

A geometrical interpretation of (43) is that $\mathcal{U}(X)$ is tangent at $X$ to the "ridge":

$$
\{X+U+V(X, G ; U): U \in \mathcal{U}(X)\}
$$

In our context we can prove that this geometrical set coincides in a neighborhood of $X$ with $\mathcal{M}_{r}$ when $G \in \operatorname{ri} \partial \lambda_{1}(X)$.

Theorem 4.6 ([33, Theorem 4.11]) Assume $(X, G) \in \mathcal{S}_{n} \times$ ri $\partial \lambda_{1}(X)$. Then there exists $\delta>0$ such that, for all $U \in \mathcal{U}(X) \cap B(0, \delta), V(X, G ; U)$ is a singleton. Moreover the map

$$
\mathcal{U}(X) \cap B(0, \delta) \ni U \mapsto X+U+V(U)
$$

is a $C^{\infty}$-parameterization of the sub-manifold $\mathcal{M}_{r}$.

This gives us a second-order development of $\lambda_{1}$ along $\mathcal{M}_{r}$. 
Theorem 4.7 ([33, Theorem 4.12-Corollary 4.13]) Take $(X, G) \in \mathcal{S}_{n} \times \operatorname{ri} \partial \lambda_{1}(X)$. Then $L_{\mathcal{U}}(X, G ; \cdot)$ is $C^{\infty}$ in a neighborhood of $U=0$ and we have the following second-order development of $\lambda_{1}$

$$
\lambda_{1}(X+U+V(U))=\lambda_{1}(X)+\langle G, U+V(U)\rangle+\frac{1}{2}\left\langle\nabla^{2} L_{\mathcal{U}}(X, G ; 0) \cdot U, U\right\rangle+o\left(\|U\|^{2}\right),
$$

where $\nabla^{2} L_{\mathcal{U}}(X, G ; 0)$ is known explicitly:

$$
\nabla^{2} L_{\mathcal{U}}(X, G ; 0)=\operatorname{proj}_{\mathcal{U}(X)}^{*} H(X, G) \operatorname{proj}_{\mathcal{U}(X)},
$$

and $H(X, G)$ is the symmetric positive semidefinite operator

$$
\mathcal{S}_{n} \ni Y \mapsto H(X, G) Y:=G Y\left[\lambda_{1}(X) I_{n}-X\right]^{\dagger}+\left[\lambda_{1}(X) I_{n}-X\right]^{\dagger} Y G .
$$

The operator $\nabla^{2} L_{\mathcal{U}}(X, G ; 0)$ is called the $\mathcal{U}$-Hessian of $\lambda_{1}$ at $(X, G)$.

\subsection{Composition with affine operator}

When composing with the affine operator $A(\cdot)$, we expect the same type of results as for $\lambda_{1}$ and we would like to have similar geometrical interpretations. It is obvious that the subspace where $f^{\prime}(x ; \cdot)$ is linear and its orthogonal complement can be written:

$$
\mathcal{U}^{f}(x)=\mathcal{A}^{-1}(\mathcal{U}(A(x))) \text { and } \mathcal{V}^{f}(x)=\mathcal{A}^{*} \mathcal{V}(A(x))
$$

Yet, when concentrating on second-order, the first difficulty encountered is that the inverse image of $\mathcal{M}_{r}$, i.e.,

$$
\left\{x \in \mathbb{R}^{m}: A(x) \in \mathcal{M}_{r}\right\}=: \mathcal{W}_{r},
$$

may be nonsmooth. Then to simplify the analysis, a transversality condition is relevant.

Definition 4.8 Transversality $W e$ say that $A(\cdot)$ is transversal to $\mathcal{M}_{r}$ at $x \in \mathcal{W}_{r}$ if and only if

$$
\mathcal{U}(A(x))+\operatorname{range} \mathcal{A}=\mathcal{S}_{n} .
$$

The transversality condition guarantees a one to one relation between $\partial f(x)$ and $\partial \lambda_{1}(A(x))$.

Lemma 4.9 Assume that transversality holds at $x \in \mathbb{R}^{m}$ and take $g \in \partial f(x)$. Then there exists a unique $G \in \partial \lambda_{1}(X)$ such that $g=\mathcal{A}^{*}(G)$; if $g$ is in ri $\partial f(x), G$ is also in ri $\partial \lambda_{1}(A(x))$. Moreover

$$
\begin{aligned}
& \operatorname{dim} \mathcal{V}^{f}(x)=\frac{r(r+1)}{2}-1, \\
& \text { and } \operatorname{dim} \mathcal{U}^{f}(x)=m+1-\frac{r(r+1)}{2} .
\end{aligned}
$$

Proof. Apply Theorem 5.5 in [33]: let $Q_{1}$ be a $n \times r$ matrix whose columns form an orthonormal basis of $E_{1}(A(x))$; then the mapping

$$
\mathcal{V}(A(x)) \ni V \mapsto \mathcal{A}^{*}(V)
$$

is nonsingular. Together with (42), this implies (46). The one-to-one relation between the subdifferentials is easily derived from the fact that $G-G^{\prime} \in \mathcal{V}(A(x))$ when $G$ and $G^{\prime}$ are in $\partial \lambda_{1}(A(x))$.

Finally transversality is a sufficient condition to obtain the differentiability of the $\mathcal{U}$-Lagrangian of $f$ and to compute its Hessian via simple chain rules. 
Theorem 4.10 ([33, Theorem 5.10]) Assume that the transversality condition holds at $x \in \mathbb{R}^{m}$ and take $g \in \operatorname{ri} \partial f(x)$. Then the $\mathcal{U}$-Lagrangian of $f$ at $x$,

$$
\mathcal{U}^{f}(x) \ni u \mapsto L_{\mathcal{U}}^{f}(x, g ; u):=\min _{v \in \mathcal{V}^{f}(x)} f(x+u+v)-g^{T} v,
$$

is $C^{\infty}$ in a neighborhood of $u=0$. In particular,

$$
\nabla L_{\mathcal{U}}^{f}(x, g ; 0)=\operatorname{proj}_{\mathcal{U} f(x)} g,
$$

and

$$
\nabla^{2} L_{\mathcal{U}}^{f}(x, g ; 0)=\operatorname{proj}_{\mathcal{U} f(x)}^{*} H^{f}(x, G) \operatorname{proj}_{\mathcal{U} f(x)},
$$

where $H^{f}(x, G):=\mathcal{A}^{*} H(A(x), G) \mathcal{A}$ and $G$ is the unique (via Lemma 4.9) vector of ri $\partial \lambda_{1}(A(x))$ such that $g=\mathcal{A}^{*}(G)$.

The operator $\nabla^{2} L_{\mathcal{U}}^{f}(x, g ; 0)$ is called the $\mathcal{U}$-Hessian of $f$ at $(x, g)$. The projection operator proj $\dot{\mathcal{U}}_{f}(x)$ is given by the expression [33, Corollary 5.7]:

$$
\operatorname{proj}_{\mathcal{U}(x)}=I_{m}-\mathcal{K}_{0}\left(\mathcal{K}_{0}^{*} \mathcal{K}_{0}\right)^{-1} \mathcal{K}_{0}^{*},
$$

where $\mathcal{K}_{0}$ is obtained by taking $\varepsilon=0$ in (37).

The transversality condition enabled M. L. Overton and R.S. Womersley, in [35], to parameterize $\mathcal{M}_{r}$ using exponentials of matrices and to transform the original unconstrained minimization problem into a smooth constrained one. This led them essentially the same second derivative formula. Going along the lines of [35] and [9], we can prove that $H(A(x), G)$ is the second covariant derivative in the Euclidean metric of the function

$$
\hat{\lambda_{1}}(M):=\frac{1}{r} \sum_{i=1}^{r} \lambda_{i}(M),
$$

which is smooth near $A(x) \in \mathcal{M}_{r}$ and coincides with $\lambda_{1}$ on $\mathcal{M}_{r}$.

Yet, note that the transversality condition is only sufficient to prove the smoothness of $L_{\mathcal{U}}^{f}(x, g ; \cdot)$. The following example shows us that it is not necessary either to get the differentiability of $L_{\mathcal{U}}^{f}(x, g ; \cdot)$ or to guarantee the smoothness of $\mathcal{W}_{r}$.

Example 4.11 Consider the mapping from $\mathbb{R}^{2}$ to $\mathcal{S}_{3}$ defined by

$$
A\left(x_{1}, x_{2}\right):=\left[\begin{array}{ccc}
x_{1}-x_{2} & 0 & 0 \\
0 & x_{2}-x_{1} & 0 \\
0 & 0 & 1
\end{array}\right]
$$

We have $f(x)=\max \left\{\left|x_{1}-x_{2}\right|, 1\right\}$ and at $\hat{x}:=\left[\begin{array}{l}0 \\ 1\end{array}\right]$ the transversality condition does not hold. We have obviously $\partial f(\hat{x})=\left\{\alpha\left[\begin{array}{c}-1 \\ 1\end{array}\right]: \alpha \in[0,1]\right\}$. Then $\mathcal{V}^{f}(0,1)=\mathbb{R}\left[\begin{array}{c}-1 \\ 1\end{array}\right]$ is unidimensional, whereas the transversality condition would imply, via Lemma $4.9, \operatorname{dim} \mathcal{V}^{f}(0,1)=2$. Yet, $\mathcal{W}_{2}$ is linear and therefore smooth in a neighborhood of $\hat{x}$ :

$$
\mathcal{W}_{2}=\hat{x}+\mathcal{U}^{f}(x)=\hat{x}+\mathbb{R}\left[\begin{array}{l}
1 \\
1
\end{array}\right]
$$

Moreover, for all $g \in \partial f(\hat{x})$ and $u \in \mathcal{U}^{f}(0,1), v(x, g ; u)=\{0\}$ and $L_{\mathcal{U}}^{f}(x, g ; u)=1$ is trivially twice differentiable. 


\section{Global second-order algorithm}

We first explain how to "stabilize" the $\mathcal{U}$-objects. Then we present the three steps of the global $\mathcal{U}$-Newton algorithm: dual, vertical and tangent steps.

\subsection{Enlargement of $\mathcal{U}$}

From the enlargement of $\partial \lambda_{1}(X)$ introduced in $\S 2.2$, we derive easily an enlargement of $\mathcal{U}(X)$. We give here the $\varepsilon$-version of Definition 4.1; note that $\sigma_{\delta_{\varepsilon}} \lambda_{1}(X)(\cdot)$ plays the role of $\lambda_{1}^{\prime}(X ; \cdot)=\sigma_{\partial \lambda_{1}(X)}(\cdot)$.

Definition 5.1 Let $\varepsilon \geq 0$ and $X \in \mathcal{S}_{n}$. We define $\mathcal{U}_{\varepsilon}(X)$ as the largest subspace where $\sigma_{\delta_{\varepsilon}} \lambda_{1}(X)$ is linear:

$$
\mathcal{U}_{\varepsilon}(X):=\left\{U \in \mathcal{S}_{n}: \sigma_{\delta_{\varepsilon} \lambda_{1}(X)}(U)+\sigma_{\delta_{\varepsilon} \lambda_{1}(X)}(-U)=0\right\}
$$

and $\mathcal{V}_{\varepsilon}(X):=\mathcal{U}_{\varepsilon}(X)^{\perp}$

Proposition 4.2 can also be extended in this context.

Proposition 5.2 Let $\varepsilon \geq 0$ and $X \in \mathcal{S}_{n}$. The subspaces $\mathcal{U}_{\varepsilon}$ and $\mathcal{V}_{\varepsilon}$ are equivalently characterized by:

(i) For any $G_{\varepsilon} \in \operatorname{ri} \delta_{\varepsilon} \lambda_{1}(X), \mathcal{U}_{\varepsilon}(X)$ and $\mathcal{V}_{\varepsilon}(X)$ are respectively the normal and tangent cones to $\delta_{\varepsilon} \lambda_{1}(X)$ at $G_{\varepsilon}$.

(ii) $\mathcal{U}_{\varepsilon}(X)$ and $\mathcal{V}_{\varepsilon}(X)$ are the subspaces respectively orthogonal and parallel to aff $\delta_{\varepsilon} \lambda_{1}(X)$.

Proposition 5.6 below will show that $\mathcal{U}_{\varepsilon}(X)$ is just the usual $\mathcal{U}$ at an appropriately shifted matrix $X_{\varepsilon}$. As a result, all the geometrical interpretations of $\S 4.1$ can be reproduced.

\subsection{Dual step}

The dual step is essentially done in $\S 3.1$ : we compute an approximation of the projection of 0 onto $\delta_{\varepsilon} f(x)$ with the support black-box method and obtain $g_{\varepsilon} \in \delta_{\varepsilon} f(x)$ such that

$$
\tilde{f}_{\varepsilon}^{\prime}\left(x ;-g_{\varepsilon}\right) \leq-\omega\left\|g_{\varepsilon}\right\|^{2} .
$$

In fact the support-black box also produces a matrix $G_{\varepsilon}$ in $\delta_{\varepsilon} \lambda_{1}(A(x))$ such that $g_{\varepsilon}=\mathcal{A}^{*}\left(G_{\varepsilon}\right)$. The subgradient $g_{\varepsilon}$ will be used to guarantee an efficient descent and the dual variable $G_{\varepsilon}$ will be needed to compute the Hessian of the $\mathcal{U}$-Lagrangian.

Actually, it is important for quadratic convergence to obtain the exact projection. The following result shows that this is possible.

Proposition 5.3 Assume $(T)$ of Definition 4.8 of and $(S C)_{0}$ of Proposition 3.1 hold at $x^{*} \in \mathbb{R}^{m}$. Then for $0<\varepsilon<\Delta_{0}\left(A\left(x^{*}\right)\right.$ ) (Definition 2.6), the $\varepsilon$-strict-complementarity condition $(S C)_{\varepsilon}$ holds in a whole neighborhood of $x^{*}$.

Proof. When $0<\varepsilon<\Delta_{0}\left(A\left(x^{*}\right)\right)$, by continuity of eigenvalues, we have $r_{\varepsilon}(x)=\operatorname{dim} E_{\varepsilon}(A(x))=r^{*}$ when $x \in B\left(x^{*}, \rho\right)$ for some $\rho>0$. In fact, for $\rho$ small enough, we have $E_{\varepsilon}(A(x))=E_{\text {tot }}(A(x))$, where $E_{\text {tot }}(A(x))$ is the total eigenspace for the $\lambda_{1}\left(A\left(x^{*}\right)\right)$-group at $A(x)$; this notion is defined in [18] and used in [41,33]. Then introducing, as in [33, Theorem 4.5], the $C^{\infty} \operatorname{map} X \mapsto Q_{t o t}(X)$ defined in a neighborhood of $A\left(x^{*}\right)$, the projection problem involved in $(S C)_{\varepsilon}$ can be written together with (10) and (26):

$$
\min _{Y \in \mathcal{C}_{r^{*}}}\left\|\mathcal{A}^{*}\left(Q_{t o t}(A(x)) Y Q_{t o t}\left(A(x)^{T}\right)\right)\right\|^{2} .
$$

Then the proof becomes similar to the one of [33, Proposition 6.9].

$\mathrm{RR} \mathrm{n}^{\circ} 3738$ 
- The first-order optimality conditions of (48) with the transversality condition at $x^{*}$, enable us to use the Implicit Function Theorem and to get a $C^{\infty}$ map $B\left(x^{*}, \delta\right) \ni x \mapsto Y(x)$ solution of (48).

- The strict complementarity condition at $x^{*}$ tells us that $Y\left(x^{*}\right)$ is positive definite.

- The continuity of $Y(\cdot)$ implies that $Y(x)$ is positive definite for $x$ in a neighborhood of $x^{*}$. This means that the $\varepsilon$-strict complementarity condition is satisfied in the latter neighborhood.

Corollary 5.4 Assume that $(T)$ and $(S C)_{0}$ hold at $x^{*}$ and take $0<\varepsilon<\Delta_{0}\left(A\left(x^{*}\right)\right)$. Then there exists $\rho_{1}>0$ such that for all $x \in B\left(x^{*}, \rho_{1}\right)$, the support-black box method with $m=1$ produces $\operatorname{proj}_{\delta_{\varepsilon}} f(x){ }^{0}$ in a finite number of steps.

Proof. Combine Proposition 3.1 with Proposition 5.3.

\subsection{Vertical step}

It would be nice to project the current point $x$ onto the manifold $\mathcal{W}_{r}:=\left\{x \in \mathbb{R}^{m}: A(x) \in \mathcal{M}_{r}\right\}$. Yet, even in the case where transversality holds, such a projection is very hard to obtain. Nevertheless, in the space of matrices it is easy to compute a point of $\mathcal{M}_{r}$ which satisfies the first-order optimality conditions associated with the projection problem. Consider a spectral decomposition of $X \in \mathcal{M}_{r}$ :

$$
X=Q_{\varepsilon} \Lambda_{\varepsilon} Q_{\varepsilon}^{T}+R_{\varepsilon} \Sigma_{\varepsilon} R_{\varepsilon}^{T},
$$

where $\Lambda_{\varepsilon}$ and $\Sigma_{\varepsilon}$ are respectively $r_{\varepsilon} \times r_{\varepsilon}$ and $\left(n-r_{\varepsilon}\right) \times\left(n-r_{\varepsilon}\right)$ diagonal matrices, and $R_{\varepsilon}$ is a $n \times\left(n-r_{\varepsilon}\right)$ matrix whose columns form an orthonormal basis of $E_{\varepsilon}(X)^{\perp}$. Here the components of $\Lambda_{\varepsilon}$ are greater than the components of $\Sigma_{\varepsilon}$. Then define for all $X \in \mathcal{S}_{n}$,

$$
\hat{\lambda}_{1, \varepsilon}(X):=\frac{1}{r_{\varepsilon}} \sum_{i=1}^{r_{\varepsilon}} \lambda_{i}(X),
$$

and

$$
X_{\varepsilon}:=\hat{\lambda}_{1, \varepsilon}(X) Q_{\varepsilon} Q_{\varepsilon}^{T}+R_{\varepsilon} \Sigma_{\varepsilon} R_{\varepsilon}^{T}
$$

We have the following result.

Theorem 5.5 The matrix $X_{\varepsilon}$ satisfies the first-order optimality conditions associated with the projection problem

$$
\min _{M \in \mathcal{M}_{r_{\varepsilon}}}\|M-X\|^{2}
$$

Proof. Let $M$ be a solution of (49). From Theorem 4.3, the manifold $\mathcal{M}_{r}$ is smooth in a neighborhood of $M$ and its normal space at $M$ is $\mathcal{V}(M)$. Then the (necessary) optimality conditions associated to (49) at $M$ are

$$
M \in \mathcal{M}_{r_{\varepsilon}} \text { and } X-M \in \mathcal{V}(M) .
$$

It is obvious that $X_{\varepsilon} \in \mathcal{M}_{r_{\varepsilon}}$, that

$$
X-X_{\varepsilon}=Q_{\varepsilon}\left[\Lambda_{\varepsilon}-\hat{\lambda}_{1, \varepsilon}(X) I_{r_{\varepsilon}}\right] Q_{\varepsilon}^{T},
$$

and that $\operatorname{tr}\left(\Lambda_{\varepsilon}-\hat{\lambda}_{1, \varepsilon}(X) I_{r_{\varepsilon}}\right)=0$. This proves $X-X_{\varepsilon} \in \mathcal{V}\left(X_{\varepsilon}\right)$ and completes the proof.

We will also use the following property. 
Proposition 5.6 Let $\varepsilon \geq 0$ and $X \in \mathcal{S}_{n}$. We have

$$
\begin{gathered}
\delta_{\varepsilon} \lambda_{1}(X)=\partial \lambda_{1}\left(X_{\varepsilon}\right) \\
\mathcal{U}_{\varepsilon}(X)=\mathcal{U}\left(X_{\varepsilon}\right) \text { and } \mathcal{V}_{\varepsilon}(X)=\mathcal{V}\left(X_{\varepsilon}\right)
\end{gathered}
$$

Proof. By construction, $\lambda_{1}\left(X_{\varepsilon}\right)=\hat{\lambda_{1 \varepsilon}}(X)$ and $E_{1}\left(X_{\varepsilon}\right)=E_{\varepsilon}(X)$. Then, together with (10) and (5), we have $\delta_{\varepsilon} \lambda_{1}(X)=\partial \lambda_{1}\left(X_{\varepsilon}\right)$. The rest is straightforward.

The step (in the space of matrices) from $A(x)$ to $A_{\varepsilon}(x):=[A(x)]_{\varepsilon}$ will be called a vertical step or $\mathcal{V}_{\varepsilon}$-step.

\subsection{Tangent step}

We assume that, at $x \in \mathbb{R}^{m}$, the dual and vertical steps have been previously computed: we have $g_{\varepsilon}(x)=\mathcal{A}^{*}\left(G_{\varepsilon}(x)\right) \in \delta_{\varepsilon} f(x)$ and $A_{\varepsilon}(x) \in \mathcal{M}_{r_{\varepsilon}}$. Then we define the following quadratic program,

$$
\left\{\begin{array}{l}
\min \left\langle G_{\varepsilon}(x), U\right\rangle+\frac{1}{2}\left\langle H\left(A_{\varepsilon}(x), G_{\varepsilon}(x)\right) U, U\right\rangle \\
U \in \mathcal{U}\left(A_{\varepsilon}(x)\right) \\
A_{\varepsilon}(x)+U \in A_{0}+\operatorname{range}(\mathcal{A})
\end{array}\right.
$$

where $H$ is defined in Theorem 4.7. When $G_{\varepsilon} \in \operatorname{ri} \delta_{\varepsilon} \lambda_{1}\left(A(x)\right.$ ), i.e., (using Proposition 5.6) $G_{\varepsilon} \in$ ri $\partial \lambda_{1}\left(A_{\varepsilon}(x)\right)$, (50) is equivalent to minimizing the second-order approximation of $L_{\mathcal{U}}\left(A_{\varepsilon}(x), G_{\varepsilon} ; \cdot\right)$ subject to $A_{\varepsilon}(x)+U$ lying in the image of the affine mapping $A(\cdot)$ : the existence of a corresponding step in the space of variables is guaranteed. Then, program (50) takes the following form in the space of variables:

$$
\left\{\begin{array}{l}
\min g_{\varepsilon}(x)^{T} d+\frac{1}{2} d^{T} H_{\varepsilon}^{f}\left(x, G_{\varepsilon}(x)\right) d \\
A(x)-A_{\varepsilon}(x)+\mathcal{A} d \in \mathcal{U}_{\varepsilon}(A(x))
\end{array}\right.
$$

To solve (51), we assume nonemptiness of the feasible domain: there exists $d_{0} \in \mathbb{R}^{m}$ such that

$$
A(x)-A_{\varepsilon}(x)+\mathcal{A} d_{0} \in \mathcal{U}_{\varepsilon}(A(x)) .
$$

When (52) is feasible, we set $u:=d-d_{0}$. Then program (51) amounts to

$$
\left\{\begin{array}{l}
\min b_{\varepsilon}(x)^{T} u+\frac{1}{2} u^{T} H^{f}\left(x, G_{\varepsilon}(x)\right) u \\
u \in \mathcal{U}_{\varepsilon}^{f}(x)
\end{array}\right.
$$

where $H^{f}$ is defined in Theorem $4.10, b_{\varepsilon}(x):=g_{\varepsilon}(x)+H^{f}\left(x, G_{\varepsilon}(x)\right) d_{0}$, and $\mathcal{U}_{\varepsilon}^{f}(x):=\left\{u \in \mathbb{R}^{m}: \mathcal{A} u \in\right.$ $\left.\mathcal{U}_{\varepsilon}(A(x))\right\}$. To guarantee that $u$ is well-defined, we assume that $H^{f}\left(x, G_{\varepsilon}(x)\right)$ is positive definite. Then, to ensure global convergence, we check whether the direction $d:=d_{0}+u$ satisfies

$$
\tilde{f}_{\varepsilon}^{\prime}(x ; d) \leq-\omega^{\prime}\left\|d^{2}\right\|
$$

where $\omega^{\prime}$ is a given number in $] 0, \omega[$.

Remark 5.7 Even when applied to a smooth function, global convergence of the Newton algorithm is an open question when the Hessian has an unbounded condition number. This explains the need for an anti-zigzag mechanism, and we found that (54) is useful to prove convergence.

\subsection{Global algorithm}

The global $\mathcal{U}$-Newton algorithm is organized as follows. 


\section{Algorithm 5.8}

Step 0 (Initialization). Choose the tolerances $\delta>0, \bar{\varepsilon}>0, \omega \in] 0,1]$ and $\left.\omega^{\prime} \in\right] 0, \omega\left[\right.$; initialize $x:=x_{0} \in$ $\mathbb{R}^{m}$ and set $\varepsilon:=\varepsilon(x)$.

Step 1 (Dual step). Compute $g_{\varepsilon}(x) \in \delta_{\varepsilon} f(x)$ and $G_{\varepsilon}(A(x)) \in \delta_{\varepsilon} \lambda_{1}(A(x))$ satisfying (47) using the support-black box method.

Step 2 (stopping criterion). If $\left\|g_{\varepsilon}(x)\right\| \leq \delta$ stop.

Step 3 (Vertical Step). Compute $A_{\varepsilon}(x)$.

Step 4 (Horizontal Step). If (52) is feasible and $H^{f}\left(x, G_{\varepsilon}(x)\right)$ is positive definite, set $d$ to the solution of (51). If (54) holds and $\|d\|>\delta$ go to Step 5.

If any of these conditions is not satisfied, set $d=-g_{\varepsilon}(x)$.

Step 5 (Line-search). Compute $t$ such that

$$
f(x+t d) \leq f(x)-\eta(x, \varepsilon),
$$

with the Line-Search of $\S 3.2$.

Step 6 (Update). Replace $x$ by $x+t d$ and $\varepsilon$ by $\varepsilon(x+t d)$; return to Step 1 .

Theorem 5.9 Assume that $f$ is bounded from below. Then Algorithm 5.8 (with $m<1$ ) stops after a finite number of iterations, yielding $\bar{x}$ satisfying the approximate minimality condition

$$
f(y) \geq f(\bar{x})-\bar{\varepsilon}-\delta\|y-\bar{x}\| \text { for all } y \in \mathbf{R}^{m} .
$$

Proof. The proof is as in Theorem 3.8, since (54) is guaranteed to hold at each iteration.

In order to obtain quadratic convergence, we introduce a condition which can be seen as the generalization of the regularity assumption needed in all Newton-type methods.

Definition 5.10 Let $x^{*} \in \mathbb{R}^{m}$ be a solution of $(P)$. We say that the Strict Second-Order Condition $(S S O C)$ holds at $x^{*}$ if $(T)$ of Definition 4.8 and $(S C)_{0}$ of Proposition 3.1 hold at $x^{*}$ and the $\mathcal{U}$-Hessian of $f$ at $\left(x^{*}, 0\right)$ is positive definite.

We first give consequences of $(S S O C)$.

Proposition 5.11 Assume that $x^{*}$ is a solution of $(P)$ and that $(S S O C)$ holds at $x^{*}$. Then

(i) $x^{*}$ is the unique solution of $(P)$,

(ii) for any $\rho>0$ there exists $\alpha>0$ such that

$$
f(x) \leq f\left(x^{*}\right)+\alpha \Rightarrow x \in B\left(x^{*}, \rho\right),
$$

(iii) for any $\rho>0$ there are $\bar{\varepsilon}$ and $\delta$ small enough, such that Algorithm 5.8 yields at least one iterate in $B\left(x^{*}, \rho\right)$, and all the subsequent iterates remain in $B\left(x^{*}, \rho\right)$ as well.

Proof. [(i)]. Decompose an arbitrary $d \in \mathbb{R}^{m}$ as $d=u+v$ with $u \in \mathcal{U}^{f}\left(x^{*}\right)$ and $v \in \mathcal{V}^{f}\left(x^{*}\right)$. By definition of the $\mathcal{U}$-Lagrangian (see Theorem 4.10), $f\left(x^{*}+d\right) \geq L_{\mathcal{U}}^{f}\left(x^{*}, 0 ; u\right)$. Hence

$$
f\left(x^{*}+d\right) \geq L_{\mathcal{U}}^{f}\left(x^{*}, 0 ; u\right)=f\left(x^{*}\right)+\frac{1}{2} u^{T} \nabla^{2} L_{\mathcal{U}}^{f}\left(x^{*}, 0 ; 0\right) u+o\left(\|u\|^{2}\right) .
$$

If $d$ is small, $u$ is small and clearly $f\left(x^{*}+d\right)>f\left(x^{*}\right)$.

$[(i i)]$. Then $\operatorname{argmin}_{x \in \mathbb{R}^{m}} f(x)$ is bounded; $f$ has bounded level sets ([16, Proposition IV.3.2.5]): say $f(x) \leq f\left(x_{0}\right)+1$ implies $\left\|x-x^{*}\right\| \leq M$. Assume for contradiction that there exist $\rho>0$ and a sequence $\left\{x_{k}\right\}_{k \in \mathbb{N}}$ such that for $k \geq 1$,

$$
f\left(x_{k}\right) \leq f\left(x^{*}\right)+\frac{1}{k} \leq f\left(x_{0}\right)+1 \text { and }\left\|x_{k}-x^{*}\right\|>\rho .
$$


Then $x_{k}$ is bounded: $\left\|x_{k}-x^{*}\right\| \leq M$. Extract a subsequence converging to some $\hat{x}$ and pass to the limit (using continuity of $f$ ) to obtain the desired contradiction:

$$
f(\hat{x}) \leq f\left(x^{*}\right) \text { and }\left\|\hat{x}-x^{*}\right\|>\rho ;
$$

$\hat{x} \neq x^{*}$ is a minimizer of $f$, which contradicts $(i)$.

$[(i i i)]$. Observe that Algorithm 5.8 produces a decreasing sequence of $f$-values: every iterate satisfies $\left\|x-x^{*}\right\| \leq M$. Given $\rho>0$, take $\alpha>0$ as in (ii), set $\bar{\varepsilon}$ and $\delta$ such that $\bar{\varepsilon}+\delta M \leq \alpha$ : from (55), at least the final iterate $\bar{x}$ satisfies $f(\bar{x}) \leq f\left(x^{*}\right)+\alpha$, hence $\bar{x} \in B\left(x^{*}, \rho\right)$; if this occurs before stopping, it occurs at each subsequent iteration.

The following lemma will enable us to guarantee that, close enough to a solution $x^{*}$, the exact multiplicity $r^{*}$ is identified.

Lemma 5.12 Assume that $0<\bar{\varepsilon}<\Delta_{0}\left(A\left(x^{*}\right)\right)$. Then, there exists $\rho_{2}>0$ such that for all $x \in$ $B\left(x^{*}, \rho_{2}\right)$,

$$
r_{\bar{\varepsilon}}(x)=\bar{r}(x)=r^{*},
$$

where $\bar{r}(x)$ is defined in (39) and $r_{\bar{\varepsilon}}(x):=\operatorname{dim} E_{\bar{\varepsilon}}(x)$.

Proof. When $0<\bar{\varepsilon}<\Delta_{0}\left(A\left(x^{*}\right)\right)$, it is clear in (39) that $r_{\bar{\varepsilon}}\left(x^{*}\right)=\bar{r}\left(x^{*}\right)=r^{*}$. Then, the result derives directly from continuity of all eigenvalues.

In the following theorem, we combine Corollary 5.4, Proposition 5.11 and Lemma 5.12 to show that after some iteration Algorithm 5.8 coincides with the $\mathcal{U}$-Newton algorithm presented in [33].

Theorem 5.13 Assume $(P)$ has a solution $x^{*} \in \mathbb{R}^{m}$ at which (SSOC) holds. Let Algorithm 5.8 be applied with $m=1$ and $\bar{\varepsilon}>0, \delta>0$ and $\left\|x_{0}-x^{*}\right\|$ sufficiently small. Assume also that the solution of (51) is accepted by Step 4 and that Step 5 produces $t=1$. Then, there exists $C>0$ such that

$$
\left\|x^{+}-x^{*}\right\| \leq C\left\|x-x^{*}\right\|^{2} .
$$

Proof. Consider $\rho_{1}>0$ of Corollary 5.4 (with $\varepsilon=\bar{\varepsilon}$ ) and $\rho_{2}>0$ of Lemma 5.12. Suppose $x_{0} \in B\left(x^{*}, \rho\right)$, with $0<\rho<\min \left\{\rho_{1}, \rho_{2}\right\}$. By Proposition $5.11(i i i)$, all subsequent iterates are in $B\left(x^{*}, \rho\right)$. Then, from Lemma 5.12, $r_{\bar{\varepsilon}}(x)=\bar{r}(x)=r^{*}$ and from Corollary 5.4 the support-black box method with $m=1$ converges in a finite number of steps. If in addition, (54) holds and the step length $t=1$ is accepted in the line-search, Algorithm 5.8 coincides with the local second order algorithm described in [33]. This algorithm has a quadratic rate of convergence [33, Theorem 6.13]: for $\rho$ small enough, there exists $C>0$ such $(56)$ when $x \in B\left(x^{*}, \rho\right)$.

In the following section we connect this work with recent results on bundle methods for semidefinite programming.

\section{From dual to proximal-type bundle methods}

When presenting the first-order method in $\S 3$, our main objectives was to provide an algorithm with a simple geometrical description to globalize second-order schemes "à la" Fletcher-Overton. Yet, for practical purposes, the following must be noted:

1. the ratio (use of the information)/(computational cost) in Algorithm 3.4 is pretty low: subgradients and $\varepsilon$-subgradients computed during the global process (Step 1) or during the local line-search are used only once,

2. the algorithm is very sensitive to the choice of the $\varepsilon$-strategy; choosing at each iteration $\varepsilon=\varepsilon(x)$ (39) ensures global convergence but may be not the best policy in practice.

Therefore there are advantages to move from a dual Markovian bundle method to a polyhedralsemidefinite proximal bundle method [23]. The obtained algorithm, in its first-order version, is closed to the one described by K.C. Kiwiel in [19] where the precision to compute the largest eigenvalue is also controlled by the global scheme.

$\mathrm{RR} \mathrm{n}^{\circ} 3738$ 
Transporting old subgradients. A first step consists in using old $\bar{\varepsilon}$-subgradients as $\varepsilon$-subgradients at the current point for some $\varepsilon \geq \bar{\varepsilon}$. This can be expressed as follows.

Proposition 6.1 Let $k>1$. Assume that, at each point $x_{i}$, we have computed $g_{i}=\mathcal{A}^{*} G_{i} \in \delta_{\bar{\varepsilon}} f\left(x_{i}\right)$ with $G_{i} \in \mathcal{C}_{n}$ for $i=1, \ldots, k-1$. Let $Q_{k}$ be an $n \times r_{k}$ matrix whose columns form an orthonormal basis of $E_{\bar{\varepsilon}}\left(A\left(x_{k}\right)\right)$. Set $\tilde{\alpha}_{k}:=\left[\alpha_{1}, \ldots, \alpha_{k-1}\right]^{T} \in \mathbb{R}^{k-1}, \mathbf{1}_{k-1} \in \mathbb{R}^{k-1}$ the vector of all ones and for $\varepsilon \geq 0$ consider the set

$$
\begin{aligned}
\mathcal{G}_{k, \varepsilon}:=\left\{\sum_{i=1}^{k-1} \alpha_{i} G_{i}+Q_{k} Y Q_{k}^{T}:\right. & \tilde{\alpha}_{k} \in \mathbb{R}_{+}^{k-1}, Y \in \mathcal{S}_{r_{k}}^{+} \\
& \mathbf{1}_{k-1}^{T} \tilde{\alpha}_{k}+\left\langle I_{r_{k}}, Y\right\rangle=1, \\
& \left.\left\langle\sum_{i=1}^{k-1} \alpha_{i} G_{i}+Q_{k} Y Q_{k}^{T}, A\left(x_{k}\right)\right\rangle \geq f\left(x_{k}\right)-\varepsilon\right\} .
\end{aligned}
$$

Then for all $\varepsilon \geq \bar{\varepsilon}$, we have

$$
\delta_{\bar{\varepsilon}} f\left(x_{k}\right) \subset A^{*} \mathcal{G}_{k, \varepsilon} \subset \partial_{\varepsilon} f\left(x_{k}\right) .
$$

Proof. This is a straightforward consequence of (7) and (25).

Inclusion (57) suggests that the convex set $\mathcal{A}^{*} \mathcal{G}_{k, \varepsilon}$ could be taken as our new approximation of $\partial_{\varepsilon} f\left(x_{k}\right)$ at $x_{k}$.

Using some duality. This leads us to a new projection problem for (35):

$$
\min \|g\|^{2}, \quad g \in \mathcal{A}^{*} \mathcal{G}_{k, \varepsilon} .
$$

Setting $\mathcal{G}_{k}:=\mathcal{G}_{k,+\infty}$ and denoting by $G(\tilde{\alpha}, Y):=\sum_{i=1}^{k-1} \alpha_{i} G_{i}+Q_{k} Y Q_{k}^{T}$ an element of $\mathcal{G}_{k}$, the projection problem amounts to

$$
\left\{\begin{array}{l}
\min \left\|\mathcal{A}^{*} G(\tilde{\alpha}, Y)\right\|^{2}, \quad G(\tilde{\alpha}, Y) \in \mathcal{G}_{k} \\
f\left(x_{k}\right)-\left\langle\sum_{i=1}^{k-1} \alpha_{i} G_{i}+Q_{k} Y Q_{k}^{T}, A\left(x_{k}\right)\right\rangle-\varepsilon \leq 0 .
\end{array}\right.
$$

Penalizing the linear inequality constraint by introducing a multiplier $2 \mu_{k}>0$, we obtain the new problem

$$
\left\{\begin{array}{l}
\min \left\|\mathcal{A}^{*} G(\tilde{\alpha}, Y)\right\|^{2}+2 \mu_{k}\left(f\left(x_{k}\right)-\left(\mathcal{A}^{*} G(\tilde{\alpha}, Y)\right)^{T} x_{k}-\left\langle A_{0}, G(\tilde{\alpha}, Y)\right\rangle-\varepsilon\right) \\
G(\tilde{\alpha}, Y) \in \mathcal{G}_{k} .
\end{array}\right.
$$

Now, multiplying the objective function by $1 /\left(2 \mu_{k}\right)$ and dropping the constant terms, it is proved in [13, Theorem 11.3.1] that (59) is exactly the dual (in the sense of Lagrangian duality [16, Chap. XII]) of the primal problem

$$
\min _{y \in \mathbb{R}^{m}} \varphi_{k}(y)+\frac{\mu_{k}}{2}\left\|y-x_{k}\right\|^{2},
$$

where $\varphi_{k}$ is the mixed polyhedral-semidefinite model of $f$ at $x_{k}$ :

$$
\varphi_{k}(y):=\max _{G \in \mathcal{G}_{k}}\left\langle A\left(x_{k}\right), G\right\rangle .
$$

Recalling the variational formulation of $\lambda_{1}$ and observing that $\mathcal{G}_{k} \supset \delta_{\varepsilon} \lambda_{1}\left(A\left(x_{k}\right)\right)$ contains at least one matrix of the form $q q^{T}$ where $q$ is a unit vector in $E_{1}\left(A\left(x_{k}\right)\right)$, it is easy to verify that this model satisfies

$$
\begin{aligned}
\varphi_{k}(y) & \leq f(y) \quad \text { for all } y \in \mathcal{R}^{m}, \\
\text { and } \varphi_{k}\left(y_{k}\right) & \geq f\left(y_{k}\right)-\varepsilon .
\end{aligned}
$$

In this approach the control parameter is $\left(\mu_{k}\right)$ which is updated with a simple strategy [20].

A contribution for large-scale problems The resulting first-order polyhedral-sdp proximal bundle method seems to be promising to solve large-scale eigenvalue problems: in [23], we present applications from control theory which involve $1000 \times 1000$ matrices depending on a large number of decision variables $m=500000$. 


\section{Dropping strict complementarity: an example from combinatorial optimization}

In this paragraph we have chosen an example from combinatorial optimization, conceived by Yu. Nesterov [29], to illustrate a qualitative distinction between interior-point methods and second-order bundle methods: for the latter methods, superlinear convergence can be observe even when sctrict complementarity does not hold. This fact seems to be corroborated by the recent theoretical work of A. Forsgren [11].

Let us consider the Boolean quadratic maximization problem

$$
(B Q M) \quad\left\{\begin{array}{l}
\max x^{T} Q x \\
x \in\{-1,1\}^{n}
\end{array}\right.
$$

where $Q=-c c^{T}$ and $c=[n-1,-1, \ldots,-1]$. A primal semidefinite relaxation [28] for $(B Q M)$ is

$$
(S D P) \quad\left\{\begin{array}{l}
\max \langle Q, X\rangle, \quad X \in \mathcal{S}_{n} \\
d(X)=\mathbf{1}_{n}, X \succeq 0
\end{array}\right.
$$

where $d(X)$ is the diagonal of $X$. The dual of this problem is then

$$
(S D P)^{*} \quad\left\{\begin{array}{l}
\min \mathbf{1}_{n}^{T} u, \quad u \in \mathbb{R}^{n} \\
D(u)-Q \succeq 0
\end{array}\right.
$$

where $D(u)$ is the matrix with $u_{1}, \ldots, u_{n}$ on its diagonal. It is easy to check that the three problems $(B Q M),(S D P)$ and $(S D P)^{*}$ have the same zero value. The solutions of $(B Q M)$ and $(S D P)^{*}$ are unique: $x^{*}=\mathbf{1}_{n}$ and $u^{*}=0 ; X^{*}=\mathbf{1}_{n} \mathbf{1}_{n}^{T}$ is a solution of $(S D P)$. Strict complementarity for $(S D P)$ [1] does not hold: the matrix $D\left(u^{*}\right)-Q+X^{*}=c c^{T}+\mathbf{1}_{n} \mathbf{1}_{n}^{T}$ is not positive definite. Consider now the equivalent eigenvalue formulation of $(S D P)^{*}[7,14]$ : making the change of variable $u=\alpha \mathbf{1}_{n}+v$ with $v^{T} \mathbf{1}_{n}=0$, we obtain

$$
\left\{\begin{array}{l}
\min n \alpha, \quad(\alpha, v) \in \mathbb{R}^{n+1} \\
\alpha I_{n} \succeq Q-D(v) \\
v^{T} \mathbf{1}_{n}=0
\end{array}\right.
$$

which is in turn equivalent to the unconstrained eigenvalue problem

$$
(E V P) \quad \min _{\tilde{v} \in \mathbb{R}^{n-1}} f(\tilde{v}),
$$

with $\mathbb{R}^{n-1} \ni \tilde{v}=\left(v_{1}, \ldots, v_{n-1}\right) \mapsto f(\tilde{v})=n \lambda_{1}\left(Q-D\left(v_{1}, \ldots, v_{n-1},-\sum_{i=1}^{n-1} v_{i}\right)\right)$. We know that the notion of strict complementarity $(S C)_{0}$ in Proposition 3.1 of an eigenvalue problem coincide with the corresponding one in semidefinite programing [33, Remark 6.6]. We can verify here by hand that $\tilde{v}^{*}=0$ and $0 \notin \operatorname{ri} \partial f(0): 0=\mathcal{A}^{*}\left(\frac{\mathbf{1}_{n} \mathbf{1}_{n}^{T}}{n}\right)$ where $\mathcal{A}^{*}$ is the linear operator

$$
\mathcal{S}_{n} \ni X \mapsto \mathcal{A}^{*} X=-n\left[\begin{array}{c}
X_{11}-X_{n n} \\
\vdots \\
X_{(n-1)(n-1)}-X_{n n}
\end{array}\right] \in \mathbb{R}^{n-1},
$$

and $\frac{\mathbf{1}_{n} \mathbf{1}_{n}^{T}}{n}$ is in the boundary of $\partial \lambda_{1}(Q)$. We run Algorithm 5.8 for $n=10$ and $\tilde{v}_{1}=\mathbf{1}_{n-1}$ with $\delta=10^{-6}, \bar{\varepsilon}=10^{-4}$ and $\omega=0.1$ and $\tilde{v}$. Figure 7 can be interpreted as follows: At the initial point the multiplicity is 1 and the distance to the second eigenvalue is large $\left(\Delta_{\bar{\varepsilon}}\left(\tilde{v}_{1}\right)=98\right)$; therefore the first-line search is very efficient (recall Theorem 2.16). Yet the descent provokes a clustering of the 9 first eigenvalues: $\Delta_{\bar{\varepsilon}}\left(\tilde{v_{k}}\right)$ become small but not enough to increase $r_{k}:=\bar{r}\left(\tilde{v}_{k}\right)$ (39) until iteration 7 where they are counted as $\frac{\bar{\varepsilon}}{n}$-eigenvalues. Then second-order steps start to be efficient and superlinear convergence is observed.

$\mathrm{RR} \mathrm{n}^{\circ} 3738$ 


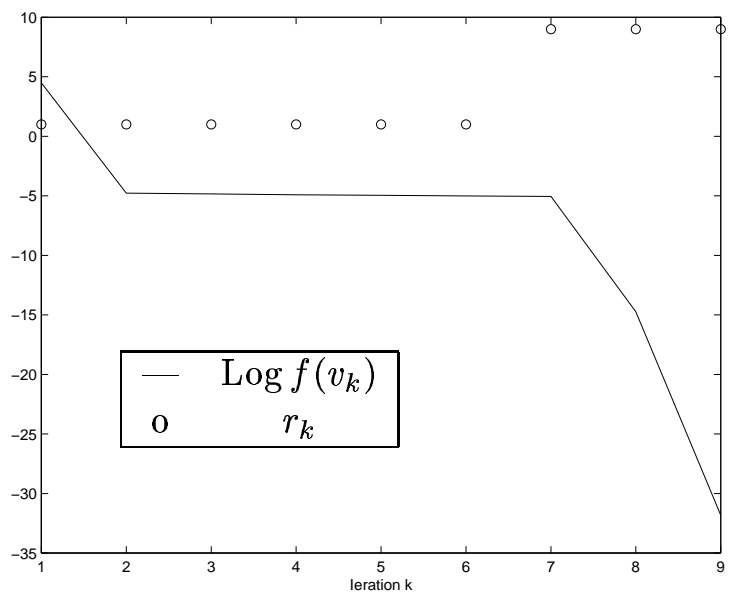

Figure 2: Superlinear convergence

\section{Conclusion}

In this paper, we have shown how to use a second-order theory, the $\mathcal{U}$-Lagrangian theory, to speed up the convergence of a first-order scheme to minimize the maximum eigenvalue function. The introduction of second-order information in a Markovian dual bundle method (the approximate eigenvalue method of $[6,37]$ ) enabled us to obtain the quadratic convergence of the resulting second-order bundle method when some regularity conditions hold. We also have made a connection with a new generation of bundle methods for semidefinite programming.

\section{Acknowledgment}

I wish to thank Claude Lemaréchal for the numerous and fruitful discussions we had together. I am also greatful to M. Overton for his careful reading and his numerous suggestions to improve the paper. Finally I thank an anonymous referee for his detailed and constructive remarks.

\section{Appendix A}

Our notation follows closely that of [24] and [15].

$\mathbb{R}^{m} m$-dimensional Euclidean space

$x^{T} y$ scalar product of $x, y \in \mathbb{R}^{m}$

$\|x\|:=\sqrt{x^{T} x}$ Euclidean norm of $x \in \mathbb{R}^{m}$

$\mathcal{U}^{\perp}$ orthogonal subspace of the subspace $\mathcal{U}$

$\operatorname{proj}_{C}: \mathbb{R}^{m} \rightarrow \mathcal{U}$ projection operator onto the closed convex set $C \subset \mathbb{R}^{m}$

aff $C$ affine hull of the nonempty set $C \subset \mathbb{R}^{m}$

ri $C$ relative interior of the convex set $C \subset \mathbb{R}^{m}$

span $C$ linear subspace generated by the nonempty set $C \subset \mathbb{R}^{m}$

$B(x, \delta)$ open ball centered at $x \in \mathbb{R}^{m}$ with radius $\delta>0$

$\mathbb{R}^{m} \ni d \mapsto \sigma_{C}(d):=\sup _{g \in C} g^{T} d$ the support function of the nonempty set $C \subset \mathbb{R}^{m}$

$F_{C}(d):=\operatorname{Argmax}_{c \in C} d^{T} c$ the face of the nonempty set $C \subset \mathbb{R}^{m}$ exposed by $d \in \mathbb{R}^{m}$

$\partial f(x)$ the subdifferential of the finite-valued convex function $f$ at $x \in \mathbb{R}^{m}$

$$
\partial f(x):=\left\{s \in \mathbb{R}^{m}: f(y)-f(x) \geq s^{T}(y-x), \text { for all } y \in \mathbb{R}^{m}\right\}
$$


$f^{\prime}(x ; d)$ the directional derivative of a convec function $f$ at $x \in \mathbb{R}^{m}$ in the direction $d \in \mathbb{R}^{m}$

$$
f^{\prime}(x ; d):=\inf _{t>0} \frac{f(x+t d)-f(x)}{t}
$$

or equivalently (see $[16, \S$ VI.1]), the support function of $\partial f(x)$

$$
f^{\prime}(x ; \cdot)=\sigma \partial f(x)^{(\cdot)}
$$

$\partial_{\varepsilon} f(x)$ the $\varepsilon$-subdifferential of $f$ at $x \in \mathbb{R}^{m}$ :

$$
\partial_{\varepsilon} f(x):=\left\{s \in \mathbb{R}^{m}: f(y)-f(x) \geq\langle s, y-x\rangle-\varepsilon \text { for all } y \in \mathbb{R}^{m}\right\}
$$

$f_{\varepsilon}^{\prime}(x ; d)$, the $\varepsilon$-directional derivative of $f$ at $x \in \mathbb{R}$ in the direction $d \in \mathbb{R}$, is the support function of $\partial_{\varepsilon} f(x)$ :

$$
f_{\varepsilon}^{\prime}(x ; \cdot):=\sigma_{\partial_{\varepsilon} f(x)}(\cdot)
$$

$\mathcal{S}_{n}$ space of $n \times n$ symmetric matrices

$\mathcal{S}_{n}^{+}$cone of positive semidefinite matrices

$X \succ Y$ (resp. $X \succeq Y$ ) means that the matrix $X-Y \in \mathcal{S}_{n}$ is positive definite (resp. positive semidefinite)

$\operatorname{tr} X:=\sum_{i=1}^{n} X_{i i}$ trace of the matrix $X \in \mathcal{S}_{n}$

$\langle X, Y\rangle:=\operatorname{tr} X Y$ Frobenius scalar product of $X, Y \in \mathcal{S}_{n}$

$\|X\|:=\sqrt{\langle X, X\rangle}$ Frobenius norm of $X \in \mathcal{S}_{n}$

$X^{\dagger}$ Moore-Penrose inverse of $X$ : if $X=\sum_{i=1}^{n} \lambda_{i}(X) q_{i} q_{i}^{T}$ is the spectral decomposition of $X, X^{\dagger}$ can be defined as $X^{\dagger}:=\sum_{\lambda_{i}(X) \neq 0} \frac{1}{\lambda_{i}(X)} q_{i} q_{i}^{T}$

$\lambda_{1}(X) \geq \ldots \geq \lambda_{n}(X)$ eigenvalues of $X \in \mathcal{S}_{n}$ in decreasing order

$E_{1}(X)$ first eigenspace of $X \in \mathcal{S}_{n}$, i.e., the eigenspace associated with $\lambda_{1}(X)$

$\mathcal{A}^{*}: \mathcal{S}_{n} \rightarrow \mathbb{R}^{m}$ is the adjoint operator of the linear operator $\mathcal{A}: \mathbb{R}^{m} \rightarrow \mathcal{S}_{n}$ defined by: for all $(d, D) \in \mathbb{R}^{m} \times \mathcal{S}_{n}$

$$
\langle D, \mathcal{A} d\rangle=d^{T} \mathcal{A}^{*} D
$$

When $\mathcal{A} d=\sum_{j=1}^{m} d_{j} A_{j}$, with $A_{j} \in \mathcal{S}_{n}$ for $j=1, \ldots, m$, we have for all $D \in \mathcal{S}_{n}, \mathcal{A}^{*} D=\left[\left\langle A_{1}, D\right\rangle, \ldots,\left\langle A_{m}, D\right\rangle\right]^{T}$

\section{References}

[1] F. Alizadeh, J.-P. A. Haeberly, and M. L. Overton. Complementarity and nondegeneracy in semidefinite programming. Mathematical Programming, 77:111-128, 1997.

[2] F. Alizadeh, J.-P. A. Haeberly, and M. L. Overton. Primal-dual interior-point methods for semidefinite programming: Convergence rates, stability and numerical results. SIAM J. Optimization, 8(3):746-768, 1998.

[3] V. I. Arnold. On matrices depending on parameters. Russian Math. Surveys, 26:29-43, 1971.

[4] R. Bellman and K. Fan. On systems of linear inequalities in Hermitian matrix variables. In V. L. Klee, editor, Convexity, volume 7 of Proceedings of Symposia in Pure Mathematics, pages 1-11. American Mathematical Society, 1963.

[5] S. Boyd, L. El Ghaoui, E. Feron, and V. Balakrishnan. Linear Matrix Inequalities in System and Control Theory, volume 15 of Studies in Applied Mathematics. SIAM, Philadelphia, PA, June 1994.

[6] J. Cullum, W. E. Donath, and P. Wolfe. The minimization of certain nondifferentiable sums of eigenvalues of symmetric matrices. Math. Programming Study, 3:35-55, 1975.

[7] C. Delorme and S. Poljak. Laplacian eigenvalues and the maximum cut problem. Mathematical Programming, 62:557-574, 1993.

$\mathrm{RR} \mathrm{n}^{\circ} 3738$ 
[8] V.F Demjanov and V.N Malozenov. Introduction to Minimax. Wiley \& Sons, 1974.

[9] A. Edelman, T. Arias, and S. T. Smith. The geometry of algorithms with orthogonality constraints. Technical report, Massachusetts Institute of Technology, Cambridge, MA 02139, February 1997. Submitted to SIAM J. Matrix. Anal. Appl.

[10] R. Fletcher. Semi-definite matrix constraints in optimization. SIAM J. Control Optim., 23:493-523, 1985.

[11] A. Forsgren. Optimality conditions for nonconvex semidefinite programming. Technical Report TRITAMAT-1998-OS6, Department of Mathematics, Royal Institute of Technology, 1998.

[12] E.G Gilbert. An iterative procedure for computing the minimum of a quadratic form on a convex set. SIAM J. Control, 4:61-80, 1966.

[13] C. Helmberg and F. Oustry. Bundle methods to minimize the maximum eigenvalue function. In Lieven Vandenberghe R. Saigal and H. Wolkovicz, editors, Hanbook on Semidefinite Programming. Theory, Algorithms and Applications. Kluwer Academic Publisher, 1999. To appear.

[14] C. Helmberg and F. Rendl. A spectral bundle method for semidefinite programming. Technical Report SC 97-37, Konrad-Zuse-Zentrum für Informationstechnik Berlin, August 1997.

[15] J.-B. Hiriart-Urruty and D. Ye. Sensivity analysis of all eigenvalues of a symmetric matrix. Numerishe Mathematik, 70:45-72, 1995.

[16] J.B. Hiriart-Urruty and C. Lemaréchal. Convex Analysis and Minimization Algorithms. Springer-Verlag, 1993. Two volumes.

[17] R. A. Horn and C. R. Johnson. Topics in Matrix Analysis. Cambridge University Press, 1991.

[18] T. Kato. Perturbation Theory for Linear Operators. Springer-Verlag, New York, 1980.

[19] K. C. Kiwiel. A linearization algorithm for optimizing control systems subject to singular value inequalities. IEEE Trans. Autom. \& Control, AC-31:595-602, 1986.

[20] K.C. Kiwiel. Proximity control in bundle methods for convex nondifferentiable minimization. Mathematical Programming, 46:105-122, 1990.

[21] M. Kojima, M. Shida, and S. Shindoh. Local convergence of predictor-corrector infeasible-interior-point algorithms for sdps and sdlcps. Mathematical Programming, 80:129-161, 1998.

[22] P. Lancaster. On eigenvalues of matrices dependent on a parameter. Numerishe Mathematik, 6:377-387, 1964.

[23] C. Lemaréchal and F. Oustry. Nonsmooth algorithms to solve semidefinite programs. In L. El Ghaoui and S-I. Niculescu, editors, Recent Advances on LMI methods in Control, Advances in Design and Control series. SIAM, 1999. To appear.

[24] C. Lemaréchal, F. Oustry, and C. Sagastizábal. The $\mathcal{U}$-Lagrangian of a convex function. Transactions of the American Mathematical Society, 1997. To appear.

[25] A. S. Lewis and M. L. Overton. Eigenvalue optimization. Acta Numerica, 5:149-190, 1996.

[26] A. Nemirovsky and P. Gahinet. The projective method for solving linear matrix inequalities. Mathematical Programming, 77:163-190, 1997.

[27] Yu. Nesterov. Interior-point methods: An old and new approach to nonlinear programming. Mathematical Programming, 79(1-3):285-297, October 1997.

[28] Yu. Nesterov. Quality of semidefinite relaxation for nonconvex quadratic optimization. CORE Discussion, Paper \# 9719, 1997.

[29] Yu Nesterov. Private communication. Center of Operations Research and Econometrics, Université Catholique de Louvain, B-1348 Louvain-La-Neuve, Belgium, May 1998.

[30] Yu. Nesterov and A. Nemirovsky. A general approach to polynomial-time algorithms design for convex programming. Technical report, Centr. Econ. \& Math. Inst., USSR Academy of Sciences, Moscow, USSR, 1988. 
[31] Yu. Nesterov and A. Nemirovsky. Interior-point polynomial methods in convex programming: Theory and applications, volume 13 of Studies in Applied Mathematics. SIAM, Philadelphia, PA, 1994.

[32] F. Oustry. Vertical developments of a convex function. Journal of Convex Analysis, 5(1):153-170, 1998.

[33] F. Oustry. The $\mathcal{U}$-Lagrangian of the maximum eigenvalue function. SIAM J. Optimization, 9(2):526-549, 1999.

[34] M. L. Overton. Large-scale optimization of eigenvalues. SIAM J. Optimization, pages 88-120, 1992.

[35] M. L. Overton and R.S. Womersley. Second derivatives for optimizing eigenvalues of symmetric matrices. SIAM J. Matrix Anal. Appl., 16(3):667-718, July 1995.

[36] M. L. Overton and X. Ye. Toward second-order methods for structured nonsmooth optimization. In S. Gomez and J-P. Hennart, editors, Advances in Optimization and Numerical Analysis, pages 97-109. Kluwer Academic Publishers, 1994.

[37] E. Polak and Y. Wardi. Nondifferentiable optimization algorithm for designing control systems having singular value inequalities. Automatica, 18:267-283, 1982.

[38] F. A. Potra and R. Sheng. A superlinearly convergent primal-dual infeasible-interior-point algorithm for semidefinite programming. SIAM J. Optimization, 8(4):1007-1028, 1998.

[39] H. Schramm and J. Zowe. A version of the bundle idea for minimizing a nonsmooth function: conceptual idea, convergence analysis, numerical results. SIAM J. Optimization, 2:121-152, 1992.

[40] L. Schwartz. Cours d'analyse, volume 1. Hermann, Paris, 1967.

[41] A. Shapiro and M. K. H Fan. On eigenvalue optimization. SIAM J. Optimization, 5(3):552-568, 1995.

[42] Lloyd N. Trefethen. Pseudospectra of linear operators. SIAM Review, 39(3):383-406, 1997.

[43] Lloyd N. Trefethen and III David Bau. Numerical Linear Algebra. SIAM, April 1997.

[44] D.Y. Ye. Sensitivity analysis of the greatest eigenvalue of a symmetric matrix via the $\varepsilon$-subdifferential of the associated convex quadratic form. Journal of Optimization Theory and Applications, 76(2), February 1993. 
Unit'e de recherche INRIA Lorraine, Technopôle de Nancy-Brabois, Campus scientifique, 615 rue du Jardin Botanique, BP 101, 54600 VILLERS LÈS NANCY

Unit'e de recherche INRIA Rennes, Irisa, Campus universitaire de Beaulieu, 35042 RENNES Cedex

Unit'e de recherche INRIA Rhône-Alpes, 655, avenue de l'Europe, 38330 MONTBONNOT ST MARTIN

Unit'e de recherche INRIA Rocquencourt, Domaine de Voluceau, Rocquencourt, BP 105, 78153 LE CHESNAY Cedex

Unit'e de recherche INRIA Sophia-Antipolis, 2004 route des Lucioles, BP 93, 06902 SOPHIA-ANTIPOLIS Cedex

\section{Éditeur}

INRIA, Domaine de Voluceau, Rocquencourt, BP 105, 78153 LE CHESNAY Cedex (France)

http://www.inria.fr

ISSN 0249-6399 